\title{
THE WEAK CONVERGENCE ANALYSIS OF TAU-LEAPING METHODS: REVISITED*
}

\author{
YUCHENG $\mathrm{HU}^{\dagger}$, TIEJUN LI ${ }^{\ddagger}$, AND BIN MIN§
}

\begin{abstract}
There are two scalings for the convergence analysis of tau-leaping methods in the literature. This paper attempts to resolve this debate in the paper. We point out the shortcomings of both scalings. We systematically develop the weak Ito-Taylor expansion based on the infinitesimal generator of the chemical kinetic system and generalize the rooted tree theory for ODEs and SDEs driven by Brownian motion to rooted directed graph theory for the jump processes. We formulate the local truncation error analysis based on the large volume scaling. We find that even in this framework the midpoint tau-leaping does not improve the weak local order for the covariance compared with the explicit tau-leaping. We propose a procedure to explain the numerical order behavior by abandoning the dependence on the volume constant $V$ from the leading error term. The numerical examples validate our arguments. We also give a general global weak convergence analysis for the explicit tau-leaping type methods in the large volume scaling.
\end{abstract}

Key words. Chemical reaction kinetics, large volume scaling, convergence analysis, rooted tree theory.

AMS subject classifications. 65C30, 65G50, 92C45.

\section{Introduction}

With the observation that biological systems are inherently random $[2,27,13,12]$, stochastic modeling and simulation of biological systems has become a very important research field in recent years. As an important tool in scientific research, numerically direct simulation of this stochastic process is an indispensable strategy to understand the behavior of microbiological systems. Gillespie's stochastic simulation algorithm (SSA) $[15,16]$ is one of the most popular algorithms. It is essentially the same as the kinetic Monte Carlo method (KMC) in the condensed matter community for simulating crystal growth [3]. Both algorithms are considered as exact simulation methods for discrete state continuous time Markov chains. The implementation of the SSA is further improved in $[14,32]$ to reduce the computational complexity.

SSA has the merit that it is exact, but since it keeps track of every reaction event, it is impractical when the reactions fire very frequently such as in the fast reversible reaction system. To speed up the simulations, Gillespie [17] proposed the tau-leaping method. By approximating the number of fired reaction events for each reaction channel with a Poisson random variable, the tau-leaping method allows us to leap along the system's history axis from one subinterval of a deterministic length $\tau$ to the next, instead of stepping along from each reaction event to the next. The idea is very appealing and proved to be very successful in numerical performance $[7,8]$. It is extended to include stiff and multiscale systems $[29,10,11]$. Some attempts to improve the accuracy of tau-leaping methods are also performed $[22,5,9]$.

${ }^{*}$ Received: March 23, 2010; accepted (in revised version): February 16, 2011. Communicated by Eric Vander-Eijnden.

${ }^{\dagger}$ Laboratory of Mathematics and Applied Mathematics and School of Mathematical Sciences, Peking University, Beijing 100871, P.R. China (huyc@pku.edu.cn).

${ }^{\ddagger}$ Laboratory of Mathematics and Applied Mathematics and School of Mathematical Sciences, Peking University, Beijing 100871, P.R. China (tieli@pku.edu.cn).

$\S$ Laboratory of Mathematics and Applied Mathematics and School of Mathematical Sciences, Peking University, Beijing 100871, P.R. China (minbmath@pku.edu.cn). 
As a promising scheme, the numerical analysis of tau-leaping has recently attracted some attention $[26,1,30]$. Actually, there is some debate on the obtained results between $[26,30]$ and [1]. The debates focus on the different scalings in the analysis. The purpose of this paper is to resolve the debates from the viewpoint of a delicate local truncation error (LTE) analysis. To be more specific, we first need some basic background.

1.1. Background. Assume that a well-stirred chemical reaction system has $N$ chemical species $\left\{S_{1}, \ldots, S_{N}\right\}$ interacting through $M$ reaction channels $\left\{R_{1}, \ldots, R_{M}\right\}$. The state of the system can be specified by the vector $\boldsymbol{X}_{t}=\left(X_{1 t}, \ldots, X_{N t}\right)^{T}$, where $X_{i t}$ denotes the number of molecules for the species $S_{i}$ at time $t$. Each reaction $R_{j}$ is characterized by its propensity function $a_{j}(\boldsymbol{x})$ and its state-change vector $\boldsymbol{\nu}_{j}=$ $\left(\nu_{1 j}, \ldots, \nu_{N j}\right)^{T},(j=1, \ldots, M)$. It is also convenient to define the total propensity $a_{0}(\boldsymbol{x})=\sum_{j=1}^{M} a_{j}(\boldsymbol{x})$. The underlying rule governing the evolution of the system is that each reaction $R_{j}$ happens with a probability of $a_{j}(\boldsymbol{x}) d t$ in the infinitesimal time $d t$, and if it happens the state of the system $\boldsymbol{X}_{t}$ will be changed by $\boldsymbol{\nu}_{j}$. With these notational conventions, the time evolution $\boldsymbol{X}_{t}$ is a Markov jump process and it obeys the CME

$$
\partial_{t} p\left(\boldsymbol{x}, t \mid \boldsymbol{x}_{0}, t_{0}\right)=\sum_{j=1}^{M} a_{j}\left(\boldsymbol{x}-\boldsymbol{\nu}_{j}\right) p\left(\boldsymbol{x}-\boldsymbol{\nu}_{j}, t \mid \boldsymbol{x}_{0}, t_{0}\right)-\sum_{j=1}^{M} a_{j}(\boldsymbol{x}) p\left(\boldsymbol{x}, t \mid \boldsymbol{x}_{0}, t_{0}\right),
$$

where $p\left(\boldsymbol{x}, t \mid \boldsymbol{x}_{0}, t_{0}\right)$ is the probability that $\boldsymbol{X}_{t}=\boldsymbol{x}$ conditioning on $\boldsymbol{X}_{t_{0}}=\boldsymbol{x}_{0}$. It is also called the Kolmogorov forward equation or Fokker-Planck equation in the probability literature.

Now the Gillespie's SSA can be implemented with the following three steps recursively:

- Step 1: sampling the waiting time $\tau$ as an exponentially distributed random variable (R.V.) with rate $a_{0}\left(\boldsymbol{X}_{t}\right)$;

- Step 2: sampling an $M$ point R.V. $k$ with probability $a_{j}\left(\boldsymbol{X}_{t}\right) / a_{0}\left(\boldsymbol{X}_{t}\right)$ for the $j$-th reaction;

- Step 3: update $\boldsymbol{X}_{t}+\tau=\boldsymbol{X}_{t}+\boldsymbol{\nu}_{k}$; then return to Step 1.

This algorithm is simple and accurate, but it will be very slow when $a_{0}\left(\boldsymbol{X}_{t}\right) \gg 1$.

The idea of tau-leaping is to choose a reasonably large $\tau$ (compared with that in the SSA) so that one can freeze the state of system and approximate the reaction numbers by Poisson random variables. So, given the state $\boldsymbol{X}_{n}$ at time $t$, the state $\boldsymbol{X}_{n+1}$ at time $t+\tau$ predicted by the tau-leaping is

$$
\boldsymbol{X}_{n+1}=\boldsymbol{X}_{n}+\sum_{j=1}^{M} \boldsymbol{\nu}_{j} \mathcal{P}_{j}\left(a_{j}\left(\boldsymbol{X}_{n}\right) \tau\right)
$$

where $\mathcal{P}_{j}\left(\lambda_{j}\right)(j=1 \ldots, M)$ are independent Poisson R.V.s with rate $\lambda_{j}$. Since this scheme resembles the explicit Euler method in numerical ODEs, we will term it Euler tau-leaping in the continued texts. With the Euler tau-leaping scheme, it can leap along the system's history axis from one subinterval of a deterministic length $\tau$ to the next, instead of firing each reaction event one by one.

1.2. Analysis of the tau-leaping method. The mathematical analysis of Euler tau-leaping method was first considered in [30]. In that paper, the authors 
built the concept of the weak local order for LTE of the moments under the scaling $\tau \rightarrow 0$, which will be termed as traditional scaling in the rest of the paper. They also obtained the explicit form of the local Taylor series for the evolution of the moments up to $\mathcal{O}\left(\tau^{2}\right)$ based on the Taylor series expansion of the waiting time probabilities. They proved that under the scaling $\tau \rightarrow 0$, global weak first order can be achieved when the propensity function $a_{j}(\boldsymbol{x})$ is linear:

$$
\left|\mathbb{E} \boldsymbol{X}_{t_{n}}^{(r)}-\mathbb{E} \boldsymbol{X}_{n}^{(r)}\right| \leq C \tau, \quad \tau \rightarrow 0,
$$

where $\mathbb{E} \boldsymbol{X}_{t_{n}}^{(r)}$ is the $r$-th moment of the exact solution $\boldsymbol{X}$ at time $t_{n}$.

The convergence result in [30] is further extended to the nonlinear propensity function case in [26]. Motivated by the theoretical analysis for diffusion processes, one of the authors proposed to analyze the convergence from the relation between the scheme and the corresponding stochastic differential equations. A mathematically equivalent description of $\boldsymbol{X}_{t}$, but which emphasizes the trajectories, can be shown to satisfy the following SDEs driven by Poisson random measure [26, 28]:

$$
d \boldsymbol{X}_{t}=\int_{0}^{A} \sum_{j=1}^{M} \boldsymbol{\nu}_{j} c_{j}\left(a ; \boldsymbol{X}_{t-}\right) \lambda(d t \times d a)
$$

where $c_{j}$ is the characteristic function

$$
c_{j}\left(a ; \boldsymbol{X}_{t}\right)= \begin{cases}1, & \text { if } a \in\left(h_{j-1}\left(\boldsymbol{X}_{t}\right), h_{j}\left(\boldsymbol{X}_{t}\right)\right], \quad j=1,2, \ldots, M, \\ 0, & \text { otherwise, }\end{cases}
$$

with $h_{j}(\boldsymbol{x})=\sum_{i=1}^{j} a_{i}(\boldsymbol{x})$, and $\lambda(d t \times d a)$ is the reference Poisson random measure associated with a Poisson point process $\left(q_{t}, t \geq 0\right)$ taking values in $[0, A]$ with the Lebesgue intensity measure $m(d t \times d a)=d t \times d a$. Here we assume that there exist such a constant $A$ that $a_{0}\left(\boldsymbol{X}_{t}\right) \leq A$ for $t \in[0, T]$. With this viewpoint, the Euler tauleaping method is exactly the explicit Euler method applied to the SDEs. Many other methods can also be constructed by comparison with the known schemes for diffusion processes. In [26], both mean square strong convergence of order $1 / 2$ and weak convergence of order 1 of the Euler tau-leaping are obtained. The authors also derived the local truncation error analysis for the midpoint tau-leaping and Poisson Runge-Kutta methods with this scaling [23].

In the practical simulations, one should consider the basic tau-leaping condition

$$
a_{0}\left(\boldsymbol{X}_{t}\right) \tau \gg 1
$$

since the tau-leaping method is only effective when (1.4) is satisfied. Otherwise one will switch to the exact SSA because the random waiting time is big enough. This is different from the numerical ODEs and diffusion processes since no exact candidate solvers exist there.

In [1], Anderson et al questioned the scaling $\tau \rightarrow 0$ in [30,26] because the condition (1.4) is ignored in the analysis. To enforce this condition, Anderson et al proposed the key assumption that there exists a volume constant $V \gg 1$ such that $\boldsymbol{X}_{t}^{V}=\boldsymbol{X}_{t} / V=$ $\boldsymbol{x}_{t} \sim \mathcal{O}(1)$ and corresponding scalings for the rate constants to ensure the large volume limit $[24,25]$. With this assumption and $\tau=V^{-\beta}$, where $0<\beta<1$, one has $\tau \rightarrow 0$ and $a_{0}\left(\boldsymbol{X}_{t}\right) \tau=\mathcal{O}\left(V^{1-\beta}\right) \rightarrow \infty$ as $V \rightarrow \infty$. Under this scaling, which will be termed as large volume scaling later on, they proved both global strong and weak convergence of the Euler tau-leaping method. 
- Strong convergence (order 1)

$$
\sup _{t \leq T} \mathbb{E}\left|\boldsymbol{X}_{t}^{V}-\boldsymbol{Z}_{t}^{V}\right| \leq C V^{-\beta}, \quad V \rightarrow \infty
$$

- Weak convergence (order 1)

$$
\sup _{t \leq T}\left|\mathbb{E} f\left(\boldsymbol{X}_{t}^{V}\right)-\mathbb{E} f\left(\boldsymbol{Z}_{t}^{V}\right)\right| \leq C V^{-\beta}, \quad V \rightarrow \infty,
$$

where $\boldsymbol{X}_{t}^{V}, \boldsymbol{Z}_{t}^{V}=\boldsymbol{Z}_{t} / V$ are rescaled variables and $\boldsymbol{Z}_{t}$ is the continuous extension of the numerical solution $\boldsymbol{X}_{n} . f$ is any continuous differentiable function. For mid-point tau-leaping, they proved that it achieves a higher order of accuracy in a strong sense and especially the second order in a weak sense.

Anderson et al's scaling indeed offers more insight on the analysis of tau-leaping, which enforces the condition (1.4) as a natural product. But we will argue in what follows that the previous analysis in $[30,26]$ is also meaningful with a suitable interpretation. At the same time, the analysis in [1] is not sufficient for explaining the realistic computations, though theoretically it is preferable. Our arguments have the following three key aspects:

1. Though the convergence results in $[30,26]$ are proved with the scaling $\tau \rightarrow 0$, the estimate (1.2) may also hold with finite size $\tau$, and this $\tau$ need not break the condition (1.4). Note that the breakdown of (1.4) is not a necessary condition to build the estimate (1.2). Similar cases appear in the numerical solution of ODEs, in which the convergence estimate is done with the scaling $\tau \rightarrow 0$, but the practical time step size criterion is obtained from the stability consideration. In this sense, the numerical order for finite $\tau$ is an extrapolation in the limit $\tau \rightarrow 0$.

2. To enforce the condition (1.4), Anderson et al considered the large volume scaling $V \rightarrow \infty$. In this limit, the rescaled variable $X_{t}^{V}$ tends to $\boldsymbol{x}(t)$, which is the solution of the reaction rate equations (RREs) and the fluctuation is not important. But in realistic examples when tau-leaping is applied, the number of molecules is usually of $\mathcal{O}\left(10^{2} \sim 10^{3}\right)$, and the large volume limit is not straightforwardly achieved. If the number of molecules is too large, one often takes the Langevin approximation or even ODE approximation [21]. To apply to the practical examples, one also needs some kind of extrapolation for finite $V$.

3. Our objective is to explain the behavior of the practical numerical examples. In the traditional numerical analysis, one detects the convergence order by measuring the error reduction compared with the step size reductions. For example, if the step size $\tau$ is halved and the error is also halved, the scheme is first order accurate. Higher reduction of the error means higher convergence order. In this procedure, the system size is fixed, which is different from the assumption made in [1]. We will show that this does not matter for the convergence order of moments, but this really makes a difference if we consider the convergence order of covariance.

In fact, the debates about the analysis of the tau-leaping methods arise because the tau-leaping methods lie on an intermediate regime. In this sense, both scalings above explore tau-leaping from two sides of a coin. In this paper, we propose to explain the computational examples by taking into account both the large volume scaling and the invariance of the system size $V$. Our results are also verified by numerical examples. 
1.3. Contribution of this paper. Theoretically we will follow the large volume scaling in this paper since it enforces the condition (1.4). But to explain the numerical behavior of the algorithm, which keeps the system size invariant, we also need the idea of extrapolation of the traditional scaling. The main contributions of this paper are as follows:

- In the scaling $\tau \rightarrow 0$, we systematically develop the weak Ito-Taylor expansion based on the infinitesimal generator of the chemical kinetic system and generalize the rooted tree theory for ODEs and diffusion processes to rooted directed graph theory for the jump processes. This structure is interesting itself and it is also the first step for the analysis of the convergence order in the large volume scaling. The essential difference between the jump process and the deterministic process is embodied in the whole local truncation error analysis. The power of the graph structure is shown in proving Theorem 2.14.

- We formulate the local truncation error analysis based on the large volume scaling. We find that though midpoint tau-leaping achieves higher local order for the moments than those of Euler tau-leaping, the local order for the covariance is not improved! This is important because the covariance is one key quantity for characterizing the random variables. For the rescaled variables in the large volume scaling, the theoretical analysis shows that the covariance is of local order $2+1 / \beta$ in $\tau$ (see Theorem 3.10), which implies that it is of global order $1+1 / \beta \geq 2$. But our numerical example shows that it is only first order accurate.

- We explain the above numerical behavior from the reason that the system size is kept invariant; the additional order $1 / \beta$ comes from the size rescaling of the volume $V$. After abandoning this effect we obtain the leading truncation order $\tau^{2}$, which suggests that the global first order conclusion is accurate though its rigorous proof is still under study. Summarizing the above procedures we claim the numerical order can be analyzed in the following 3 steps.

- Step 1. Write down the local truncation error estimate in the traditional scaling $\tau \rightarrow 0$;

- Step 2. Reorder the error terms according to the magnitude of the power of $V$ under the large volume scaling;

- Step 3. Abandon the dependence on $V$ in the leading order terms and find the order $p$ on $\tau$, which is the weak local order.

- We generalize the global weak convergence results in [1] to general higher order estimates in the large volume scaling. But our new framework establishes the global weak convergence of an explicit tau-leaping method based on the argument that the local truncation estimates imply global convergence (stability is satisfied automatically for explicit one-step methods). This relieves the algorithm designer from the abstract analysis by just considering the local truncation errors.

1.4. Paper outline. The remainder of this paper is arranged as follows. In Section 2 we will develop the rooted directed graph theory to represent the weak Taylor expansion for chemical kinetic systems, which is applied to derive the expansion of the moments and covariance. The power of the graph restructure is shown in proving Theorem 2.14. In Section 3 we will perform local truncation analysis for Euler tauleaping and midpoint tau-leaping in the large volume scaling. Upon using the rooted directed graph representation, we get a sharp estimate for covariance which reveals 
that midpoint tau-leaping is no more accurate than Euler tau-leaping. In Section 4, we give the global weak convergence estimate for a general explicit tau-leaping type method in the large volume scaling. In Section 5 , we inspect a numerical example to compare the numerical behavior of some existing tau-leaping methods. The rooted tree theory for numerical ODEs and diffusion processes is attached in the Appendix.

\section{Weak Taylor expansion for chemical reaction systems}

In this section, we aim to use graphical representation to perform weak Taylor expansion for chemical kinetic systems in the scaling $\tau \rightarrow 0$. This idea was first proposed by Butcher for ODEs [6] and is nicely reviewed in [20], where the Taylor expansion is intuitively represented through the growth of rooted trees. Rossler generalized this theory to SDEs driven by Brownian motion in [31]. In his theory he introduced the colored rooted tree to describe the effect of the Brownian noise. A concise review for both the rooted tree and the colored rooted tree theory is presented in the Appendix. Our analysis shows that we have to use the rooted directed graph structure rather than the tree structure to describe the weak Ito-Taylor expansion for the chemical kinetic system. We should remark here that the concept "rooted directed graph" is a bit confusing since the "root" node does not make any sense in a graph in general. With a more rigorous statement we mean the uni-source directed acyclic graph (USDAG) structure as defined in Definition 2.4. In this sense, we call the unique source as our "root", and the USDAG as the "rooted directed graph". This "rooted directed graph" structure can be used as a systematic tool to investigate the order of schemes, and inspire people to construct higher order numerical simulation algorithms for chemical kinetic systems in future works. This is also the first step towards formulating the local truncation error analysis in the large volume scaling.

2.1. Rooted directed graph representation for the weak Taylor expansions. At first let us recall the infinitesimal generator and the weak Taylor expansion for the chemical kinetic systems without proof. The readers may refer to [18] for more details.

Lemma 2.1 (Infinitesimal generator for the chemical kinetic system). Assume that $\boldsymbol{X}_{t}$ satisfies (1.3) and $f(\boldsymbol{x}) \in C\left(\mathbb{R}^{N}\right)$. Then the following equation holds:

$$
\mathbb{E} f\left(\boldsymbol{X}_{t}\right)-\mathbb{E} f\left(\boldsymbol{X}_{0}\right)=\mathbb{E} \int_{0}^{t} \mathcal{L} f\left(\boldsymbol{X}_{s}\right) d s,
$$

where $\mathcal{L}$ is the infinitesimal generator for the chemical kinetic system

$$
\mathcal{L} f(\boldsymbol{x})=\lim _{t \rightarrow 0} \frac{\mathbb{E}^{0, \boldsymbol{x}} f\left(\boldsymbol{X}_{t}\right)-f(\boldsymbol{x})}{t}=\sum_{i=1}^{M} a_{i}(\boldsymbol{x})\left(f\left(\boldsymbol{x}+\boldsymbol{\nu}_{i}\right)-f(\boldsymbol{x})\right) .
$$

Based on the above lemma, we immediately obtain the following Ito-Taylor expansion.

Lemma 2.2 (Weak Ito-Taylor expansion). Assume $\boldsymbol{X}_{t}$ satisfies (1.3) and $f(\boldsymbol{x}) \in$ $C\left(\mathbb{R}^{N}\right)$. Then we have the following expansion:

$$
\mathbb{E}^{t, \boldsymbol{x}} f\left(\boldsymbol{X}_{t+h}\right)=\sum_{n=0}^{r} \frac{h^{n}}{n !} \mathcal{L}^{(n)} f(\boldsymbol{x})+\mathcal{O}\left(h^{r+1}\right),
$$

where $\mathcal{L}^{(0)} f(\boldsymbol{x})=f(\boldsymbol{x}), \mathcal{L}^{(n)} f(\boldsymbol{x})=\mathcal{L}\left(\mathcal{L}^{(n-1)} f(\boldsymbol{x})\right), n=1,2, \ldots, r$. 
In principle, to derive the local truncation errors for a numerical scheme, the Equation (2.3) can be applied directly to get all of the terms up to the needed order. With this viewpoint, the truncation error analysis is just a straightforward computation. But as observed by Butcher for numerical ODEs [6], this procedure can be nicely described in a more visual way, that is, through the rooted tree structure. In this section, we generalize this visual representation for chemical kinetic systems in the spirit of Butcher. Another generalization for the diffusion processes is pursued by Rössler in [31]. We would like to emphasize that this representation is not a game but a practical tool in the later analysis, which is embodied in Section 2.2. This visualization is also transparent, and clarifies the qualitative difference between deterministic ODEs, diffusion processes driven by Brownian motion, and the chemical kinetic jump processes.

Below we will take index notations similar to those used in ODEs and diffusion processes. We define the difference

$$
f^{j}(\boldsymbol{x})=f\left(\boldsymbol{x}+\boldsymbol{\nu}_{j}\right)-f(\boldsymbol{x})
$$

with respect to the $j$-th reaction for any continuous function $f(\boldsymbol{x})$. Then formally we have

$$
f^{j k}(\boldsymbol{x})=f\left(\boldsymbol{x}+\boldsymbol{\nu}_{j}+\boldsymbol{\nu}_{k}\right)-f\left(\boldsymbol{x}+\boldsymbol{\nu}_{j}\right)-f\left(\boldsymbol{x}+\boldsymbol{\nu}_{k}\right)+f(\boldsymbol{x}) .
$$

We can formulate the index notations for each term in (2.3), which we will only list the cases for $n=1,2$ in what follows.

- When $n=1$, we have the index representation for the infinitesimal generator of the chemical kinetic system

$$
\mathcal{L}^{(1)} f(\boldsymbol{x})=\mathcal{L} f(\boldsymbol{x})=a_{i} f^{i}(\boldsymbol{x})
$$

by the Einstein summation convention.

- When $n=2$, we should consider the product rule for the operator $\mathcal{L}$. The straightforward calculation shows

$$
\mathcal{L}(f g)=\sum_{i=1}^{M} a_{i} f^{i} g^{i}+\sum_{i=1}^{M} a_{i} f^{i} g+\sum_{i=1}^{M} a_{i} g^{i} f .
$$

We still simply denote it as

$$
\mathcal{L}(f g)=a_{i} f^{i} g^{i}+a_{i} f^{i} g+a_{i} g^{i} f
$$

with Einstein's summation convention, though we note that the index $i$ appears three times in the first term in (2.6). In fact, that is the difference between the jump process and the deterministic or diffusion processes. With the product rule (2.7), or applying the infinitesimal generator to the function $f$ twice, we obtain

$$
\mathcal{L}^{(2)} f=a_{i} a_{j} f^{i j}+a_{j} a_{i}^{j} f^{i}+a_{j} a_{i}^{j} f^{i j} .
$$

Indeed the formula (2.7) is a general tool for larger $n$ cases. We call the terms appearing in (2.5) and (2.8) elementary differences, which is parallel to the concept of elementary differentials for the ODEs and diffusion processes. They can be represented by the rooted directed graph as shown in Figure 2.1. 


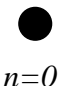

$n=0$
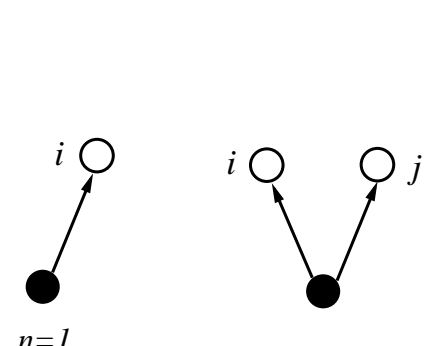
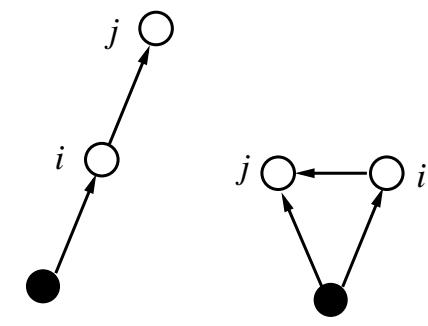

FIGURE 2.1. The rooted directed graph representation of the elementary differences for SDEs driven by the state dependent Poisson noises when $n=0,1,2$. The solid circle in $n=2$ case represents the root node, which corresponds to the function $f$ in (2.8). Each empty circle represents one reaction type, which corresponds to a coefficient function a. The directed graph grows upwards when $n$ increases. Each connecting arrow for a pair of nodes represents a "Superior-Inferior" relation directed from the superior node to the inferior node, which corresponds to take a superscript operation to the Superior node. Note that here the superscript operation $i$ represents the function change after the $i$-th reaction fires. It is not taking the derivative with respect to the $i$-th variable as in the last two subsections.

Now let us generalize the rooted directed graph theory to $n>2$ cases. We have the general product rule for the operator $\mathcal{L}$.

Proposition 2.3. For general $n \in \mathbb{N}$, the following expansion holds:

$$
\mathcal{L}\left(f_{1} f_{2} \ldots f_{n}\right)=\sum_{k=1}^{n} \sum_{|S|=k} a_{j} \prod_{u \in S} f_{u}^{j} \prod_{v \in S^{c}} f_{v}
$$

Here $S \subseteq\{1,2, \ldots, n\}$ and the Einstein summation convention is applied.

Proof. The proof is a direct induction by applying the Equation (2.7) recursively.

Remark 2.1. According to the Proposition 2.3, the number of terms after the action of the infinitesimal generator of the chemical kinetic system to one product of $n$-terms grows with the rate $2^{n}-1$. This is very different from that of the ODEs with the order $n$.

To state the general weak Ito-Taylor expansion similar to those for ODEs and SDEs driven by Brownian motion, we need the following concepts borrowed from graph theory [19].

DEFINITION 2.4. The following notions are standard in directed graph theory.

1. Ordered pair.

An ordered pair is a pair of nodes with an order associated with them. If the two nodes are $i$ and $j$, then we denote this ordered pair as $i \rightarrow j$ or $j \rightarrow i$. In general they are different because of the order.

2. Binary relation.

$A$ binary relation from a set $\mathscr{A}$ to a set $\mathscr{B}$ is a set of ordered pairs $i \rightarrow j$ where $i$ is an element of $\mathscr{A}$ and $j$ is an element of $\mathscr{B}$. When $\mathscr{A}=\mathscr{B}$, we call it a binary relation on $\mathscr{A}$. 
3. Superior and Inferior sets.

For a graph $\boldsymbol{g}=(\mathscr{A}, \mathcal{E})$, where $\mathscr{A}$ is the set of nodes and $\mathcal{E}$ is the binary relation on $\mathscr{A}$, we denote the Superior-set of $i \in \mathscr{A}$ as $\mathbb{S}(i)=\left\{j_{1}, \ldots, j_{m}\right\} \subset \mathscr{A}$ if the ordered pairs $j_{k} \rightarrow i(k=1, \ldots, m)$ belong to the binary relation $\mathcal{E}$, and the Inferior-set of $i \in \mathscr{A}$ as $\mathbb{I}(i)=\left\{j_{1}, \ldots, j_{m}\right\} \subset \mathscr{A}$ if the ordered pairs $i \rightarrow j_{k}(k=$ $1, \ldots, m)$ belong to the binary relation $\mathcal{E}$. If the node $i$ does not have Superiors or Inferiors, we will define $\mathbb{S}(i)$ or $\mathbb{I}(i)$ as empty set $\phi$, respectively.

4. Directed acyclic graph.

A directed acyclic graph is a graph $\boldsymbol{g}=(\mathscr{A}, \mathcal{E})$ where $\mathscr{A}$ is the set of nodes and $\mathcal{E}$ is the binary relation on $\mathscr{A}$ with the property that there are no directed cycles in it.

5. Degree, source, and sink.

The outdegree of $i \in \mathscr{A}$ in a directed graph $\boldsymbol{g}=(\mathscr{A}, \mathcal{E})$ is the times that $i$ appears in ordered pairs $i \rightarrow j, j \neq i$. The indegree of $i \in \mathscr{A}$ in a directed graph $\boldsymbol{g}=(\mathscr{A}, \mathcal{E})$ is the times that $i$ appears in ordered pairs $j \rightarrow i, j \neq i$. When the indegree or outdegree of $i$ equals 0 , we call the node $i$ a source or a sink of the directed graph, respectively.

Definition 2.5 (Labeled uni-source directed acyclic graph (LUSDAG)). Suppose $\mathscr{A}$ is an ordered chain of indices $\mathscr{A}=\{R<i<j<k<l<\cdots\}$ and $\#(\mathscr{A})=q \geq 1$. A labeled uni-source directed acyclic graph (LUSDAG) $\boldsymbol{g}=\left(\mathscr{A}, \mathcal{E}_{\boldsymbol{g}}\right)$ satisfies the following conditions:

1. $\boldsymbol{g}$ is a directed acyclic graph;

2. The node $\{R\}$ is the unique source, which is also called "root" in accordance with the concept in numerical ODEs;

3. for every ordered pair $i_{1} \rightarrow i_{2} \in \mathcal{E}_{\boldsymbol{g}}\left(i_{1}, i_{2} \in \mathscr{A}\right), i_{1}<i_{2}$.

The order of a LUSDAG $\boldsymbol{g}$ is equal to the number of its nodes, which is denoted by $\rho(\boldsymbol{g})=q$. The number of edges of a LUSDAG $\boldsymbol{g}$ is denoted as $\gamma(\boldsymbol{g})$. The set of all $L U S D A G s$ of order $q$ is denoted by $\mathcal{L G}_{q}$.
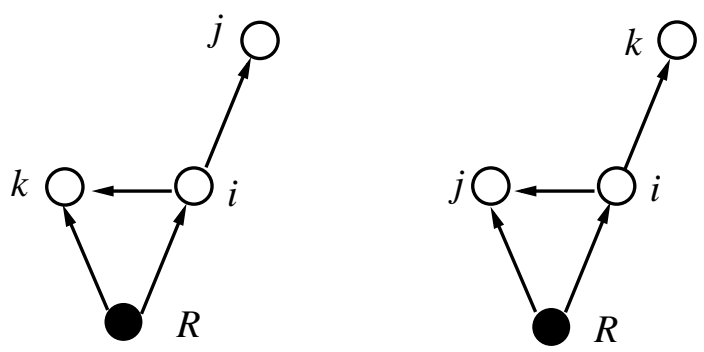

Figure 2.2. The labeled uni-source directed acyclic graph. Here $\rho(\boldsymbol{g})=q=4$ for both graphs. Each node is named with an index from the set $\mathscr{A}$. With the notations for LUSDAG, the left figure corresponds to the elementary difference $a_{i}^{j} a_{j} a_{k} f^{i, k}$; the right figure corresponds to the elementary difference $a_{i}^{k} a_{j} a_{k} f^{i, j}$. These two graphs are equivalent in the sense of Definition 2.7.

The above definition can be schematically shown in Figure 2.2, where $\rho(\boldsymbol{g})=q=4$. Every LUSDAG $\boldsymbol{g} \in \mathcal{L G}_{q}$ is associated with an elementary difference.

Definition 2.6 (Elementary difference). For a $L U S D A G \boldsymbol{g} \in \mathcal{L G}_{q}$ we call

$$
f(\boldsymbol{g})(\boldsymbol{x})=a_{i_{1}}^{\mathbb{I}\left(i_{1}\right)} \cdots a_{i_{q-1}}^{\mathbb{I}\left(i_{q-1}\right)} f^{\mathbb{I}(R)}
$$


its corresponding elementary difference. The summand is a product of $(q-1)$ a's and one $f$, where the lower index $i_{k}(k=1, \ldots, q-1)$ of each a corresponds to each node of $\boldsymbol{g}$ except the root, and the upper indices of it are its corresponding Inferiors. If $\mathbb{I}(i)=\phi$, the upper index of a will be left empty correspondingly. The upper indices of $f$ run through all of the Inferiors of the root. Here we have used the Einstein summation convention. Two examples are shown in Figure 2.2.

Definition 2.7 (Equivalence of LUSDAGs). Two LUSDAGs $\boldsymbol{g}=\left(\mathscr{A}, \mathcal{E}_{\boldsymbol{g}}\right), \boldsymbol{h}=$ $\left(\mathscr{A}, \mathcal{E}_{\boldsymbol{h}}\right) \in \mathcal{L G}_{q}$ are equivalent if there exists a permutation $\sigma: \mathscr{A} \rightarrow \mathscr{A}$, such that $\sigma(R)=R$ and $\mathbb{S}_{\boldsymbol{g}} \sigma=\sigma \mathbb{S}_{\boldsymbol{h}}$ on $\mathscr{A} \backslash\{R\}$, where $\mathbb{S}_{\boldsymbol{g}}$ and $\mathbb{S}_{\boldsymbol{h}}$ are the Superior-set finding operators defined in Definition 2.4 for $\boldsymbol{g}$ and $\boldsymbol{h}$, respectively.

Definition 2.8 (Uni-source directed acyclic graph (USDAG)). An equivalence class of q-th order LUSDAG is called a USDAG of order $q$. The set of all this type of graphs of order $q$ is denoted by $\mathcal{G}_{q}$. The order of a USDAG $\boldsymbol{g} \in \mathcal{G}_{q}$ is defined as the order of a representative and is again denoted by $\rho(\boldsymbol{g})$. Furthermore we denote by $\alpha(\boldsymbol{g})$ (for $\boldsymbol{g} \in \mathcal{G}_{q}$ ) the number of elements in the equivalence class $\boldsymbol{g}$.

Definition $2.9\left(\mathcal{T} \mathcal{G}_{q}\right.$ as the subset of $\left.\mathcal{G}_{q}\right)$. Define the subset of $\mathcal{G}_{q}$ which includes $\boldsymbol{g}$ satisfying $\rho(\boldsymbol{g})=\gamma(\boldsymbol{g})+1$ as $\mathcal{T} \mathcal{G}_{q}$.

It is straightforward to see that $\mathcal{T} \mathcal{G}_{q}$ can be mapped one-to-one to $\mathcal{T}_{q}$ (see Appendix A), which includes all rooted trees in ODE satisfying $\rho(\boldsymbol{t})=q$. This fact is very important in that we can use facts in ODE to investigate the properties of $\mathcal{T} \mathcal{G}_{q}$. It is also straightforward to observe the following fact.

Proposition 2.10 (Equivalence of the definition of $\alpha(\boldsymbol{g})$ ). If $\boldsymbol{g} \in \mathcal{T} \mathcal{G}_{q}$, then the multiplicity function $\alpha(\boldsymbol{g})$ is equal to $\alpha(\boldsymbol{g})$ when $\boldsymbol{g}$ is viewed as an object in $\mathcal{T}_{q}$ (refer to Appendix A for the definition).

We can now state the weak Ito-Taylor expansion of the exact solution with unisource directed acyclic graph representation.

Theorem 2.11 (Weak Ito-Taylor expansion with rooted directed graph notations). Assume $\boldsymbol{X}_{t}$ satisfies (1.3) and $f(\boldsymbol{x}) \in C\left(\mathbb{R}^{N}\right)$. Then we have the following expansion:

$$
\begin{aligned}
\mathbb{E}^{t, \boldsymbol{x}} f\left(\boldsymbol{X}_{t+h}\right) & =\sum_{q=1}^{r+1} \sum_{\boldsymbol{g} \in \mathcal{L} \mathcal{G}_{q}} \frac{h^{\rho(\boldsymbol{g})-1}}{(\rho(\boldsymbol{g})-1) !} f(\boldsymbol{g})(\boldsymbol{x})+\mathcal{O}\left(h^{r+1}\right) \\
& =\sum_{q=1}^{r+1} \sum_{\boldsymbol{g} \in \mathcal{G}_{q}} \frac{h^{\rho(\boldsymbol{g})-1}}{(\rho(\boldsymbol{g})-1) !} \alpha(\boldsymbol{g}) f(\boldsymbol{g})(\boldsymbol{x})+\mathcal{O}\left(h^{r+1}\right) .
\end{aligned}
$$

Proof. The theorem is true for $q=1,2,3$ by a simple comparison between Figure 2.1 and the equations (2.5) and (2.8). Suppose that it holds for $q$. To prove it holds for $q+1$ case, we first observe that each elementary difference term is different and complete in $\mathcal{L G}_{q}$. Then the action of the operator $\mathcal{L}$ to each elementary difference term will generate $2^{q}-1$ terms according to the product rule (2.9). For each new term $a_{j} \prod_{u \in S} f_{u}^{j} \prod_{v \in S^{c}} f_{v}$ it corresponds to generating a new link from the index $u \in S$ to a new index $j$ which is listed after all of the previous indices in the ordered index set $\mathscr{A}$ in the graphical representation. These graphs traverse all of the possibilities from one node, two nodes, ..., in $\mathcal{L G}_{q}$ to $j$, so it is clear that all labeled uni-source directed acyclic graphs of order $q+1$ appear in the coefficients of the $h^{q+1}$ term, each of them 
exactly once. Grouping together the terms with identical elementary differences, we have the second expression in (2.11).

\subsection{Moment expansion based on the rooted directed graph theory.}

By using the rooted directed graph theory, we can obtain the weak Taylor expansion for any function to arbitrarily high order. Especially, using the facts in rooted tree theory for ODEs, we can get more details about the expansion of covariance, which is the main object in the next section.

The following proposition is the direct result of applying rooted directed graph for each component of $\boldsymbol{x}^{(r)}$, the $r$-fold tensor product for the vector $\boldsymbol{x}$.

Proposition 2.12 (The $\mathcal{O}\left(\tau^{3}\right)$ local expansion for the moments). If $\boldsymbol{X}_{t}$ satisfies Equation (1.3), then we have

$$
\begin{aligned}
\mathbb{E}^{t, \boldsymbol{x}} \boldsymbol{X}_{t+\tau}^{(r)}= & \boldsymbol{x}^{(r)}+\tau a_{j}\left[\boldsymbol{x}^{(r)}\right]^{j}+\frac{\tau^{2}}{2} a_{j} a_{k}\left[\boldsymbol{x}^{(r)}\right]^{j k}+\frac{\tau^{2}}{2} a_{j}^{k} a_{k}\left[\boldsymbol{x}^{(r)}\right]^{j}+\frac{\tau^{2}}{2} a_{j}^{k} a_{k}\left[\boldsymbol{x}^{(r)}\right]^{j k} \\
& +\frac{\tau^{3}}{6} a_{j} a_{k} a_{l}\left[\boldsymbol{x}^{(r)}\right]^{j k l}+\frac{\tau^{3}}{2} a_{j}^{k} a_{k} a_{l}\left[\boldsymbol{x}^{(r)}\right]^{j l} \\
& +\frac{\tau^{3}}{6} a_{j}^{k} a_{k}^{l} a_{l}\left[\boldsymbol{x}^{(r)}\right]^{j}+\frac{\tau^{3}}{6} a_{j}^{k l} a_{k} a_{l}\left[\boldsymbol{x}^{(r)}\right]^{j} \\
& +\frac{\tau^{3}}{6} \sum_{\boldsymbol{g} \in \mathcal{G}_{4}, \gamma(\boldsymbol{g}) \geq \rho(\boldsymbol{g})} \alpha(\boldsymbol{g}) \boldsymbol{x}^{(r)}(\boldsymbol{g})+\mathcal{O}\left(\tau^{4}\right)
\end{aligned}
$$

for the $r$-th moments. Here the superscript for $j, k$, or $l$ takes the definition in (2.4).

When $r=1, \boldsymbol{x}^{j k}$ will be zero! So it may be relatively easy to obtain the higher order expansions for the mean. We have the following proposition by straightforwardly using the rooted directed graph expansion for $f(\boldsymbol{x})=\boldsymbol{x}$.

Proposition 2.13. If $\boldsymbol{X}_{t}$ satisfies Equation (1.3), then

$$
\begin{aligned}
\mathbb{E}^{t, \boldsymbol{x}} \boldsymbol{X}_{t+\tau}= & \boldsymbol{x}+\tau a_{j} \boldsymbol{\nu}_{j}+\frac{\tau^{2}}{2} a_{j}^{k} a_{k} \boldsymbol{\nu}_{j} \\
& +\frac{\tau^{3}}{6} a_{j}^{k l} a_{k} a_{l} \boldsymbol{\nu}_{j}+\frac{\tau^{3}}{6} a_{j}^{k} a_{k}^{l} a_{l} \boldsymbol{\nu}_{j}+\frac{\tau^{3}}{6} a_{j}^{k l} a_{k}^{l} a_{l} \boldsymbol{\nu}_{j} \\
& +\mathcal{O}\left(\tau^{4}\right),
\end{aligned}
$$

where the three $\mathcal{O}\left(\tau^{3}\right)$ terms can be represented by rooted directed graphs as in Figure 2.3.

Now let us address the issue about the expansion of the covariance. The following theorem precisely describes the weak expansion of the covariance and will play an important role in the next section.

Theorem 2.14. Assume that $\boldsymbol{X}_{t}$ satisfies Equation (1.3). Then the expansion coefficients of the $\tau^{q}(q \geq 1)$ terms of $\operatorname{Cov}\left(\boldsymbol{X}_{t+\tau}\right)$ must take the form

$$
a_{i_{1}}^{\mathbb{I}\left(i_{1}\right)} \cdots a_{i_{q}}^{\mathbb{I I}\left(i_{q}\right)} \boldsymbol{\nu}_{j_{1}} \boldsymbol{\nu}_{j_{2}}
$$

where we have dropped the superscript $\{t, \boldsymbol{x}\}$ of the covariance function Cov for simplicity. Define the characteristic number

$$
\lambda_{q}:=\kappa_{q}-q+2,
$$



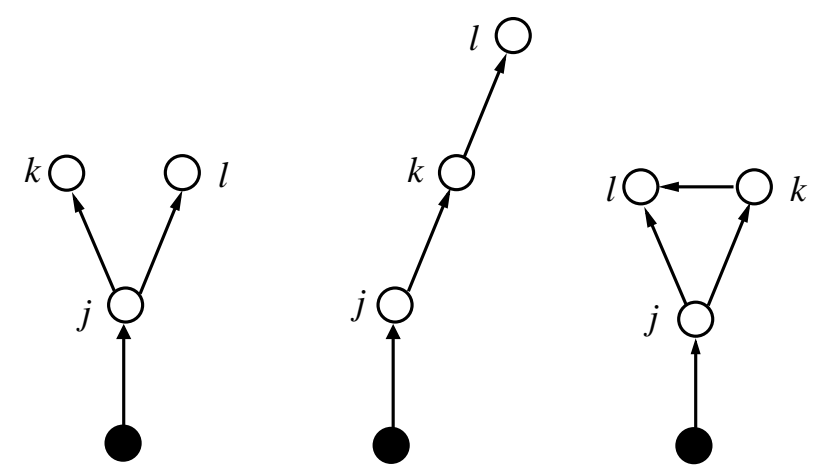

Figure 2.3. The rooted directed graph representations for three $\mathcal{O}\left(\tau^{3}\right)$ terms for the mean. There are much fewer terms here since many of them are zeros because of the linearity of the function $\boldsymbol{f}(\boldsymbol{x})=\boldsymbol{x}$.

where $\kappa_{q}=\sum_{k=1}^{q} \#\left(\mathbb{I}\left(i_{k}\right)\right)$ is the number of superscripts in the a terms in (2.14). Then we have

$$
\lambda_{q} \geq 1
$$

For $q=1,2$ the corresponding error terms are

$$
\tau a_{j} \boldsymbol{\nu}_{j}^{(2)}, \quad \frac{\tau^{2}}{2} a_{j}^{k} a_{k} \boldsymbol{\nu}_{j}^{(2)}+\frac{\tau^{2}}{2} a_{j}^{k} a_{k}\left(\boldsymbol{\nu}_{j} \boldsymbol{\nu}_{k}+\boldsymbol{\nu}_{k} \boldsymbol{\nu}_{j}\right) .
$$

Proof. By applying the weak Ito-Taylor expansion (2.11) to each component of $f(\boldsymbol{x})=\boldsymbol{x}^{(2)}$ and $f(\boldsymbol{x})=\boldsymbol{x}$, we obtain that the coefficient of $\tau^{q}(q \geq 1)$ of the expansion of $\operatorname{Cov}\left(\boldsymbol{X}_{t+\tau}\right)$ has the following form:

$$
\sum_{\boldsymbol{g} \in \mathcal{G}_{q+1}} \frac{\alpha(\boldsymbol{g})}{(\rho(\boldsymbol{g})-1) !} \boldsymbol{x}^{(2)}(\boldsymbol{g})-\sum_{\substack{q_{1}+q_{2}=q \\ q_{1}, q_{2} \geq 0}} \sum_{\boldsymbol{g}_{1} \in \mathcal{G}_{2} \in \mathcal{G}_{q_{2}+1}+1} \frac{\alpha\left(\boldsymbol{g}_{1}\right)}{\left(\rho\left(\boldsymbol{g}_{1}\right)-1\right) !} \frac{\alpha\left(\boldsymbol{g}_{2}\right)}{\left(\rho\left(\boldsymbol{g}_{2}\right)-1\right) !} \boldsymbol{x}\left(\boldsymbol{g}_{1}\right) \boldsymbol{x}\left(\boldsymbol{g}_{2}\right) .
$$

To prove the desired results, we separate the terms in the first summation in (2.15) to two cases which are schematically shown in Figures 2.4 and 2.5. We will consider these two cases one by one.

Case 1: There are two Inferiors for the root node (Figure 2.4).

At first, we have the identity

$$
\left[\boldsymbol{x}^{(2)}\right]^{i j}=\boldsymbol{\nu}_{i} \boldsymbol{\nu}_{j}+\boldsymbol{\nu}_{j} \boldsymbol{\nu}_{i}, \boldsymbol{x}^{i}=\boldsymbol{\nu}_{i}
$$

If the graph $\boldsymbol{g}$ displays a tree structure, we have

$$
\lambda_{q}=(\rho(\boldsymbol{g})-3)-q+2=0 .
$$

Otherwise $\lambda_{q} \geq 1$. When $\boldsymbol{g}$ has the tree structure we denote it as

$$
\boldsymbol{g}=\left[\hat{\boldsymbol{g}}_{1}, \hat{\boldsymbol{g}}_{2}\right]
$$



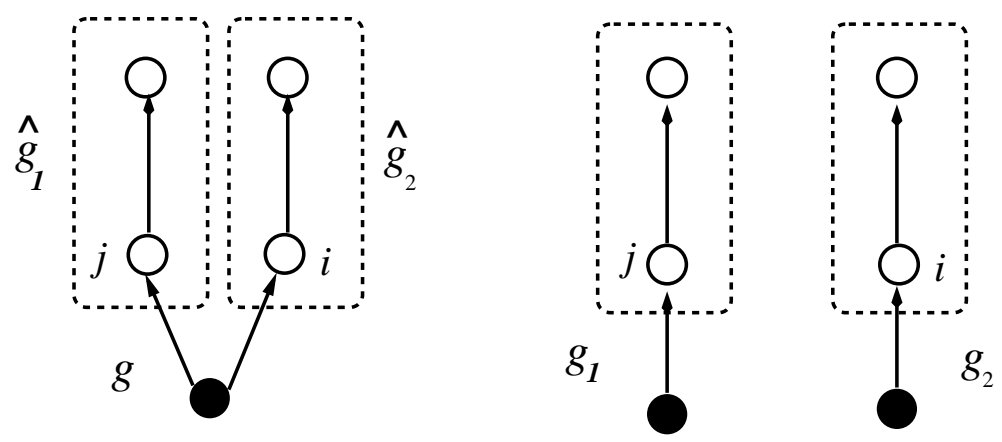

FiguRE 2.4. The schematics of the rooted directed graph for Case 1. In the left part of the figure $\boldsymbol{g}$ corresponds to the graph in the first term of (2.15), in the right part of the figure $\boldsymbol{g}_{1}, \boldsymbol{g}_{2}$ correspond to the graphs in the second term of (2.15).

by borrowing the tree notation in Appendix A, where $\hat{\boldsymbol{g}}_{1}, \hat{\boldsymbol{g}}_{2}$ are two branches of the graph $\boldsymbol{g}$ after cutting the root node. Thanks to (A.8) and Proposition 2.10 we have

$$
\frac{\alpha(\boldsymbol{g})}{(\rho(\boldsymbol{g})-1) !}=\frac{\alpha\left(\hat{\boldsymbol{g}}_{1}\right)}{\rho\left(\hat{\boldsymbol{g}}_{1}\right) !} \frac{\alpha\left(\hat{\boldsymbol{g}}_{2}\right)}{\rho\left(\hat{\boldsymbol{g}}_{2}\right) !} \frac{1}{\mu !}=\frac{\alpha\left(\boldsymbol{g}_{1}\right)}{\left(\rho\left(\boldsymbol{g}_{1}\right)-1\right) !} \frac{\alpha\left(\boldsymbol{g}_{2}\right)}{\left(\rho\left(\boldsymbol{g}_{2}\right)-1\right) !} \frac{1}{\mu !},
$$

where

$$
\boldsymbol{g}_{1}=\left[\hat{\boldsymbol{g}}_{1}\right], \boldsymbol{g}_{2}=\left[\hat{\boldsymbol{g}}_{2}\right] .
$$

When $\hat{\boldsymbol{g}}_{1} \neq \hat{\boldsymbol{g}}_{2}$, we have $\mu=1$ and

$$
\boldsymbol{x}^{(2)}(\boldsymbol{g})=\left(\boldsymbol{\nu}_{i} \boldsymbol{\nu}_{j}+\boldsymbol{\nu}_{j} \boldsymbol{\nu}_{i}\right) a_{i}\left(\hat{\boldsymbol{g}}_{1}\right) a_{j}\left(\hat{\boldsymbol{g}}_{2}\right)=\boldsymbol{x}\left(\boldsymbol{g}_{1}\right) \boldsymbol{x}\left(\boldsymbol{g}_{2}\right)+\boldsymbol{x}\left(\boldsymbol{g}_{2}\right) \boldsymbol{x}\left(\boldsymbol{g}_{1}\right) .
$$

When $\hat{\boldsymbol{g}}_{1}=\hat{\boldsymbol{g}}_{2}$, we have $\mu=2$ and thus

$$
\frac{1}{2} \boldsymbol{x}^{(2)}(\boldsymbol{g})=\frac{1}{2}\left(\boldsymbol{\nu}_{i} \boldsymbol{\nu}_{j}+\boldsymbol{\nu}_{j} \boldsymbol{\nu}_{i}\right) a_{i}\left(\hat{\boldsymbol{g}}_{1}\right) a_{j}\left(\hat{\boldsymbol{g}}_{1}\right)=\boldsymbol{x}\left(\boldsymbol{g}_{1}\right) \boldsymbol{x}\left(\boldsymbol{g}_{1}\right)
$$

To summarize, in Case 1 all the terms of the first summation in (2.15) with $\lambda_{q}=0$ exactly cancel with the corresponding terms in the second summation.
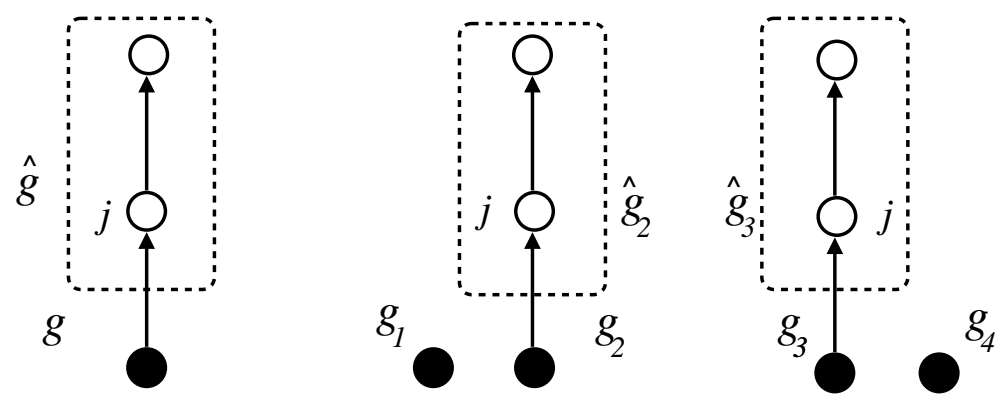

Figure 2.5. The schematics of the rooted directed graph for Case 2. In the left part of the figure $\boldsymbol{g}$ corresponds to the graph in the first term of (2.15), and in the right parts of the figure $\left(\boldsymbol{g}_{1}, \boldsymbol{g}_{2}\right),\left(\boldsymbol{g}_{3}, \boldsymbol{g}_{4}\right)$ correspond to two possibilities for the graphs in the second term of (2.15). 
Case 2: There is only one Inferior for the root node (Figure 2.5).

We have the identity

$$
\left[\boldsymbol{x}^{(2)}\right]^{j}=\boldsymbol{x} \boldsymbol{\nu}_{j}+\boldsymbol{\nu}_{j} \boldsymbol{x}+\boldsymbol{\nu}_{j} \boldsymbol{\nu}_{j}
$$

For the last term, we have

$$
\lambda_{q} \geq(\rho(\boldsymbol{g})-2)-q+2=1,
$$

and the identity only holds when $\boldsymbol{g}$ is a tree. So only the first two terms interesting. For the second summation in (2.15), we consider the cases $q_{1}=0, q_{2}=q$ or $q_{1}=q, q_{2}=0$. It is not difficult to derive the following identities from Figure 2.5:

$$
\begin{aligned}
\boldsymbol{x}^{(2)}(\boldsymbol{g}) & =\left(\boldsymbol{x} \boldsymbol{\nu}_{j}+\boldsymbol{\nu}_{j} \boldsymbol{x}\right) a_{j}(\hat{\boldsymbol{g}})=\boldsymbol{x}\left(\boldsymbol{g}_{1}\right) \boldsymbol{\nu}_{j} a_{j}\left(\hat{\boldsymbol{g}}_{2}\right)+a_{j}\left(\hat{\boldsymbol{g}}_{3}\right) \boldsymbol{\nu}_{j} \boldsymbol{x}\left(\boldsymbol{g}_{4}\right) \\
& =\boldsymbol{x}\left(\boldsymbol{g}_{1}\right) \boldsymbol{x}\left(\boldsymbol{g}_{2}\right)+\boldsymbol{x}\left(\boldsymbol{g}_{3}\right) \boldsymbol{x}\left(\boldsymbol{g}_{4}\right) .
\end{aligned}
$$

The coefficients of these terms are also the same. These facts show that in Case 2 the cancellation for the first summation with $\lambda_{q}=0$ ( $\boldsymbol{g}$ is a tree in this case) also holds.

For the second summation in (2.15), only the pattern corresponding to the right part of the Figure 2.4 exists because of cancellation. A calculation shows

$$
\lambda_{q} \geq\left(q_{1}-1\right)+\left(q_{2}-1\right)-q+2=0 .
$$

and the identity only holds when $\boldsymbol{g}_{1}$ and $\boldsymbol{g}_{2}$ both have tree structure. But these terms also disappear because of cancellation!

When $q=1,2$, the error terms can be derived by direct calculations. The proof is complete.

\section{Local truncation error analysis in large volume scaling}

In this section we will perform the local weak consistency analysis for Euler tauleaping and midpoint tau-leaping in the large volume scaling. Though the global weak convergence for the rescaled variables has been proved in [1], the LTE analysis has the merit that it is more transparent and instructive for constructing new methods. It is also the first step for analyzing the global weak convergence order. By using the rooted directed graph representation developed in the previous section, we get a sharp estimate for covariance which reveals that midpoint tau-leaping is no more accurate than Euler tau-leaping. This is also consistent with our numerical results in the next section. Since the covariance is more important than the second moment about the origin to characterize the fluctuation behavior of the random variables, it also motivates us to explore weakly higher order methods in future studies. Note also that the analysis for the covariance is not included in [1].

3.1. Notations and backgrounds. To perform the weak consistency analysis for tau-leaping methods in the large volume scaling, we borrow some notations from [1]. Let $\mathbb{L}^{V}=\left\{\boldsymbol{y} \mid \boldsymbol{y}=\boldsymbol{X} / V, \boldsymbol{X} \in \mathbb{Z}^{N}\right\}$. We also make the same assumptions as introduced in [1].

Assumption 3.1. The following basic assumptions are needed in the large volume scaling:

1. State variables $\boldsymbol{X}_{t}=\mathcal{O}(V)$ for $i=1, \ldots, N$ where $V \gg 1$. 
2. Propensities $a_{j}\left(\boldsymbol{X}_{t}\right)(j=1, \ldots, M)$ are $\mathcal{O}(V)$ such that the following scaling holds:

$$
a_{j}\left(\boldsymbol{X}_{t}\right)=V a_{j}^{V}\left(\boldsymbol{X}_{t}^{V}\right), \quad \boldsymbol{X}_{t}^{V}=\boldsymbol{X}_{t} / V \sim \mathcal{O}(1) .
$$

For simplicity we will denote $\boldsymbol{x}=\boldsymbol{X}_{t}^{V}$ in the later text. The rescaled propensities $a_{j}^{V}(\boldsymbol{x})$ satisfies the continued condition.

3. $a_{j}^{V}(\boldsymbol{x})(j=1, \ldots, M)$ are $C_{0}^{\infty}$ functions with compact support $\Omega$ with respect to $\boldsymbol{x}$, and thus satisfy

$$
\sup _{\boldsymbol{x} \in \mathbb{R}^{N},|\alpha|<\infty}\left|D^{\alpha} a_{j}^{V}(\boldsymbol{x})\right| \leq L_{j},
$$

where $\alpha \in \mathbb{N}^{N}$.

One key property of the above scaling is if we define

$$
\tau=1 / V^{\beta}, 0<\beta<1,
$$

then we have

$$
\tau \sum_{j} a_{j}\left(\boldsymbol{X}_{t}\right)=1 / V^{\beta} V \sum_{k} a_{j}^{V}(\boldsymbol{x})=\mathcal{O}\left(V^{1-\beta}\right) \gg 1,(V \gg 1),
$$

which satisfies the tau-leaping condition automatically.

Definition 3.1 (Difference in the large volume scaling). Let us define the difference notation in the large volume scaling as

$$
f^{V, j}(\boldsymbol{x})=V\left(f\left(\boldsymbol{x}+\boldsymbol{\nu}_{j} / V\right)-f(\boldsymbol{x})\right)
$$

for $\boldsymbol{x} \in \mathbb{L}^{V}$, and recursively define

$$
\begin{aligned}
f^{V, j k}(\boldsymbol{x}) & =\left(f^{V, j}\right)^{V, k}(\boldsymbol{x}) \\
& =V^{2}\left(f\left(\boldsymbol{x}+\boldsymbol{\nu}_{j} / V+\boldsymbol{\nu}_{k} / V\right)-f\left(\boldsymbol{x}+\boldsymbol{\nu}_{j} / V\right)-f\left(\boldsymbol{x}+\boldsymbol{\nu}_{k} / V\right)+f(\boldsymbol{x})\right)
\end{aligned}
$$

and higher order differences.

REMARK 3.2. We should remark here that though notationally $\left(a_{j}\right)^{V, k}(\boldsymbol{x})$ and $\left(a_{j}^{V}\right)^{k}(\boldsymbol{x})$ look similar, they are indeed different since

$$
\left(a_{j}\right)^{V, k}(\boldsymbol{x})=V\left(a_{j}\left(\boldsymbol{x}+\boldsymbol{\nu}_{k} / V\right)-a_{j}(\boldsymbol{x})\right)
$$

and

$$
\left(a_{j}^{V}\right)^{k}(\boldsymbol{x})=a_{j}^{V}\left(\boldsymbol{x}+\boldsymbol{\nu}_{k}\right)-a_{j}^{V}(\boldsymbol{x})=V^{-1}\left(a_{j}\left(V \boldsymbol{x}+V \boldsymbol{\nu}_{k}\right)-a_{j}(V \boldsymbol{x})\right) .
$$

But in fact this confusion will never appear because when we use the traditional difference notation $(2.4), \boldsymbol{x}$ must be the primitive variable in $\mathbb{N}^{N}$; while when we use the large volume difference notation (3.4), $\boldsymbol{x}$ must have been transformed to the rescaled variable in $\mathbb{L}^{V}$. With this convention in mind, both notations $\left(a_{j}\right)^{V, k}(\boldsymbol{x})$ and $\left(a_{j}^{V}\right)^{k}(\boldsymbol{x})$ do not make sense. For notational convenience, we will simply take

$$
a_{j}^{V, k}:=\left(a_{j}^{V}\right)^{V, k}, a_{j}^{V, k l}:=\left(a_{j}^{V}\right)^{V, k l}, \ldots
$$


in the later texts.

We notice that when the rescaled variable $\boldsymbol{x}=\boldsymbol{X}_{t} / V \sim \mathcal{O}(1)$, which is independent of $V$, then

$$
\left[\boldsymbol{x}^{(r)}\right]^{V, j}=\mathcal{O}(1), \quad\left[\boldsymbol{x}^{(r)}\right]^{V, j k}=\mathcal{O}(1), \ldots
$$

Similar results also hold for $(f(\boldsymbol{x}))^{V, j k}$, etc., when $f$ is a rescaled function which is independent of $V$.

To analyze the convergence of tau-leaping, it is natural to introduce a continuous extension of the numerical solution

$$
d \boldsymbol{Y}_{t}=\int_{0}^{A} \sum_{j=1}^{M} \boldsymbol{\nu}_{j} c_{j}(a ; \boldsymbol{Y} \circ \eta(t)) \lambda(d t \times d a),
$$

where $\eta(s)=t_{n}$ if $t_{n} \leq s<t_{n+1}$. For the midpoint tau-leaping method also proposed by Gillespie [17]

$$
\left\{\begin{array}{l}
\boldsymbol{X}_{n+\frac{1}{2}}=\boldsymbol{X}_{n}+\sum_{k=1}^{M} \boldsymbol{\nu}_{k} \frac{1}{2} \tau a_{k}\left(\boldsymbol{X}_{n}\right) \\
\boldsymbol{X}_{n+1}=\boldsymbol{X}_{n}+\sum_{k=1}^{M} \boldsymbol{\nu}_{k} \mathcal{P}_{k}\left(a_{k}\left(\boldsymbol{X}_{n+\frac{1}{2}}\right), \tau\right)
\end{array}\right.
$$

the corresponding continuous extension may be taken as

$$
d \boldsymbol{Z}_{t}=\int_{0}^{A} \sum_{j=1}^{M} \boldsymbol{\nu}_{j} c_{j}(a ; \boldsymbol{\mu} \circ \boldsymbol{Z} \circ \eta(t)) \lambda(d t \times d a),
$$

where

$$
\boldsymbol{\mu}(\boldsymbol{z})=\boldsymbol{z}+\frac{1}{2} \tau \sum_{i} a_{i}(\boldsymbol{z}) \boldsymbol{\nu}_{i}
$$

In [1], Anderson et al carried out the error analysis for Euler tau-leaping and midpoint tau-leaping with the rescaled variables

$$
\boldsymbol{X}_{t}^{V} \triangleq \boldsymbol{X}_{t} / V, \boldsymbol{Y}_{t}^{V} \triangleq \boldsymbol{Y}_{t} / V, \boldsymbol{Z}_{t}^{V} \triangleq \boldsymbol{Z}_{t} / V
$$

In the large volume scaling they got the following global weak convergence results:

Theorem 3.2 (Anderson, Ganguly, Kurtz). For any $f \in C_{0}^{2}\left(\mathbb{R}^{N}\right)$, there exist constants $C_{1}=C_{1}(f, T)>0, C_{2}=C_{2}(f, T)>0$ such that

$$
\begin{gathered}
V^{\beta}\left|\mathbb{E} f\left(\boldsymbol{X}_{T}^{V}\right)-\mathbb{E} f\left(\boldsymbol{Y}_{T}^{V}\right)\right| \leq C_{1}, \\
V^{2 \beta}\left|\mathbb{E} f\left(\boldsymbol{X}_{T}^{V}\right)-\mathbb{E} f\left(\boldsymbol{Z}_{T}^{V}\right)\right| \leq C_{2} .
\end{gathered}
$$

In the following context we will use the "rooted directed graph" expansion to discuss the local version of the estimates for all moments about the origin and especially the covariance. 


\subsection{Local truncation errors for the moments about the origin.}

LEMMA 3.3 (Transformation from traditional scaling to large volume scaling). We have the relation

$$
f(\boldsymbol{g})\left(\boldsymbol{X}_{t}\right) \sim \mathcal{O}\left(V^{\rho(\boldsymbol{g})-\gamma(\boldsymbol{g})}\right)
$$

for each elementary difference as in (2.10) with the function $f\left(\boldsymbol{X}_{t}\right)=V f^{V}(\boldsymbol{x})$ and $f^{V}(\boldsymbol{x}) \sim \mathcal{O}(1)$ which is independent of $V$. Thus

$$
f(\boldsymbol{g})\left(\boldsymbol{X}_{t}\right) \sim \mathcal{O}(V)
$$

if $\boldsymbol{g} \in \mathcal{T} \mathcal{G}_{q}$ and

$$
f(\boldsymbol{g})\left(\boldsymbol{X}_{t}\right) \sim \mathcal{O}\left(V^{p}\right), p \leq 0 \text { and } p \in \mathbb{Z}
$$

if $\boldsymbol{g} \in \mathcal{G}_{q} \backslash \mathcal{T} \mathcal{G}_{q}$.

Proof. The proof can be obtained by the straightforward application of Equations (3.4) and (3.5), the convention (3.6) and the transformation $\boldsymbol{x}=\boldsymbol{X}_{t} / V$. We have

$$
a_{j}^{\mathbb{I}(j))}=V^{1-\#(\mathbb{I}(j))} a_{j}^{V, \mathbb{I}(j)}, \quad f^{\mathbb{I}(R)}=V^{1-\#(\mathbb{I}(j))} f^{V, \mathbb{I}(R)} .
$$

Thus the power of $V$ is

$$
\sum_{j=1}^{q-1}\left(1-\#\left(\mathbb{I}\left(i_{j}\right)\right)\right)+1-\#(\mathbb{I}(R))=q-\gamma(\boldsymbol{g})=\rho(\boldsymbol{g})-\gamma(\boldsymbol{g})
$$

for any $\boldsymbol{g} \in \mathcal{G}_{q}$.

Lemma 3.4. If $\boldsymbol{X}_{t}$ satisfies Equation (1.3) and $\boldsymbol{x}=\boldsymbol{X}_{t}^{V}=\boldsymbol{X}_{t} / V$, then

$$
\begin{aligned}
\mathbb{E}^{t, \boldsymbol{x}}\left[\boldsymbol{X}_{t+\tau}^{V}\right]^{(r)}= & \boldsymbol{x}^{(r)}+\tau a_{j}^{V}\left[\boldsymbol{x}^{(r)}\right]^{V, j}+\frac{\tau^{2}}{2} a_{j}^{V} a_{k}^{V}\left[\boldsymbol{x}^{(r)}\right]^{V, j k} \\
& +\frac{\tau^{2}}{2} a_{j}^{V, k} a_{k}^{V}\left[\boldsymbol{x}^{(r)}\right]^{V, j}+\frac{\tau^{2}}{2} V^{-1} a_{j}^{V, k} a_{k}^{V}\left[\boldsymbol{x}^{(r)}\right]^{V, j k} \\
& +\frac{\tau^{3}}{6} a_{j}^{V} a_{k}^{V} a_{l}^{V}\left[\boldsymbol{x}^{(r)}\right]^{V, j k l}+\frac{\tau^{3}}{2} a_{j}^{V, k} a_{k}^{V} a_{l}^{V}\left[\boldsymbol{x}^{(r)}\right]^{V, j l} \\
& +\frac{\tau^{3}}{6} a_{j}^{V, k} a_{k}^{V, l} a_{l}^{V}\left[\boldsymbol{x}^{(r)}\right]^{V, j}+\frac{\tau^{3}}{6} a_{j}^{V, k l} a_{k}^{V} a_{l}^{V}\left[\boldsymbol{x}^{(r)}\right]^{V, j} \\
& +\mathcal{O}\left(V^{-4 \beta}\right),
\end{aligned}
$$

where the notation $\mathbb{E}^{t, \boldsymbol{x}}$ means the expectation with respect to the initial state $\boldsymbol{x}$ at time $t$ for the rescaled process $\boldsymbol{X}_{t}^{V}$.

Proof. Straightforward application of Lemma 3.3 and the fact

$$
\left(\boldsymbol{X}_{t}^{(r)}\right)^{\mathbb{I}(R)}=V^{r-\#(\mathbb{I}(R))}\left(\boldsymbol{x}^{(r)}\right)^{V, \mathbb{I}(R)}
$$

gives

$$
\left(\boldsymbol{X}_{t}^{(r)}\right)(\boldsymbol{g}) \sim \mathcal{O}\left(V^{r-1+\rho(\boldsymbol{g})-\gamma(\boldsymbol{g})}\right)
$$


for each elementary difference as in (2.10) with the root label $R$. We get (3.15) by combining (2.12), the fact $\beta<1$, and the above result.

For the LTE estimate of Euler tau-leaping methods, we have

Lemma 3.5. If $\boldsymbol{Y}_{t}$ satisfies Equation (3.8), then

$$
\begin{aligned}
\mathbb{E}^{t, \boldsymbol{x}}\left[\boldsymbol{Y}_{t+\tau}^{V}\right]^{(r)}= & \boldsymbol{x}^{(r)}+\tau a_{j}^{V}\left[\boldsymbol{x}^{(r)}\right]^{V, j}+\frac{\tau^{2}}{2} a_{j}^{V} a_{k}^{V}\left[\boldsymbol{x}^{(r)}\right]^{V, j k} \\
& +\frac{\tau^{3}}{6} a_{j}^{V} a_{k}^{V} a_{l}^{V}\left[\boldsymbol{x}^{(r)}\right]^{V, j k l}+\mathcal{O}\left(V^{-4 \beta}\right) .
\end{aligned}
$$

Proof. From the continuous extension of tau-leaping methods (3.8), we obtain an expansion similar to Equation (2.12) except that the jumping rate $a_{k}$ should be constant. So all the terms including the difference of $a_{k}$ will disappear. The proof is completed.

For the LTE estimate of midpoint tau-leaping methods, we have

Lemma 3.6. If $\boldsymbol{Z}_{t}$ satisfies Equation (3.10), then

$$
\begin{aligned}
\mathbb{E}^{t, \boldsymbol{x}}\left[\boldsymbol{Z}_{t+\tau}^{V}\right]^{(r)}= & \boldsymbol{x}^{(r)}+\tau a_{j}^{V}\left[\boldsymbol{x}^{(r)}\right]^{V, j}+\frac{\tau^{2}}{2} \hat{a}_{j}^{V, k} a_{k}^{V}\left[\boldsymbol{x}^{(r)}\right]^{V, j} \\
& +\frac{\tau^{2}}{2} a_{j}^{V} a_{k}^{V}\left[\boldsymbol{x}^{(r)}\right]^{V, j k}+\frac{\tau^{3}}{6} a_{j}^{V} a_{k}^{V} a_{l}^{V}\left[\boldsymbol{x}^{(r)}\right]^{V, j k l} \\
& +\frac{\tau^{3}}{2} a_{j}^{V} \hat{a}_{k}^{V, l} a_{l}^{V}\left[\boldsymbol{x}^{(r)}\right]^{V, j k}+\frac{\tau^{3}}{8} \hat{a}_{j}^{V, k l} a_{k}^{V} a_{l}^{V}\left[\boldsymbol{x}^{(r)}\right]^{V, j} \\
& +\mathcal{O}\left(V^{-4 \beta}\right),
\end{aligned}
$$

where

$$
\hat{a}_{j}^{V, k}:=\sum_{p} \frac{\partial a_{j}^{V}}{\partial x_{p}} \nu_{k p}, \quad \hat{a}_{j}^{V, k l}:=\sum_{p, q} \frac{\partial^{2} a_{j}^{V}}{\partial x_{p} \partial x_{q}} \nu_{k p} \nu_{l q} .
$$

Proof. Based on arguments similar to those in Lemma 3.5, we get

$$
\begin{aligned}
\mathbb{E}^{t, \boldsymbol{x}}\left[\boldsymbol{Z}_{t+\tau}^{V}\right]^{(r)} & =\boldsymbol{x}^{(r)}+\tau a_{j}^{V}\left(\boldsymbol{\mu}^{V}(\boldsymbol{x})\right)\left[\boldsymbol{x}^{(r)}\right]^{V, j}+\frac{\tau^{2}}{2} a_{j}^{V}\left(\boldsymbol{\mu}^{V}(\boldsymbol{x})\right) a_{k}^{V}\left(\boldsymbol{\mu}^{V}(\boldsymbol{x})\right)\left[\boldsymbol{x}^{(r)}\right]^{V, j k} \\
& +\frac{\tau^{3}}{6} a_{j}^{V}\left(\boldsymbol{\mu}^{V}(\boldsymbol{x})\right) a_{k}^{V}\left(\boldsymbol{\mu}^{V}(\boldsymbol{x})\right) a_{l}^{V}\left(\boldsymbol{\mu}^{V}(\boldsymbol{x})\right)\left[\boldsymbol{x}^{(r)}\right]^{V, j k l}+\mathcal{O}\left(V^{-4 \beta}\right),
\end{aligned}
$$

where $\boldsymbol{\mu}^{V}(\boldsymbol{x})$ is the rescaled version of $\boldsymbol{\mu}(\boldsymbol{x})$ defined as

$$
\boldsymbol{\mu}^{V}(\boldsymbol{x})=\boldsymbol{x}+\frac{1}{2} \tau \sum_{k} a_{k}^{V}(\boldsymbol{x}) \boldsymbol{\nu}_{k}
$$

For $a_{j}^{V}\left(\boldsymbol{\mu}^{V}(\boldsymbol{x})\right)$, we have

$$
a_{j}^{V}\left(\boldsymbol{\mu}^{V}(\boldsymbol{x})\right)=a_{j}^{V}(\boldsymbol{x})+\frac{1}{2} \tau \hat{a}_{j}^{V, k} a_{k}^{V}+\frac{1}{8} \tau^{2} \hat{a}_{j}^{V, k l} a_{k}^{V} a_{l}^{V}+\mathcal{O}\left(V^{-3 \beta}\right) .
$$

Substituting $a_{j}^{V}\left(\boldsymbol{\mu}^{V}(\boldsymbol{x})\right)$ to $(3.21)$ and noticing that $\left[\boldsymbol{x}^{(r)}\right]^{V, j k}=\left[\boldsymbol{x}^{(r)}\right]^{V, k j}$, we get the desired result. 
Now we can obtain the LTE for the moments about the origin for Euler tau-leaping and midpoint tau-leaping.

Proposition 3.7 (LTE for the moments of origin). If $\boldsymbol{X}_{t}, \boldsymbol{Y}_{t}$, and $\boldsymbol{Z}_{t}$ satisfy the Equations (1.3), (3.8), and (3.10) respectively, then

$$
\begin{aligned}
& \left|\mathbb{E}^{t, \boldsymbol{x}}\left[\boldsymbol{X}_{t+\tau}^{V}\right]^{(r)}-\mathbb{E}^{t, \boldsymbol{x}}\left[\boldsymbol{Y}_{t+\tau}^{V}\right]^{(r)}\right|=\mathcal{O}\left(V^{-2 \beta}\right), \\
& \left|\mathbb{E}^{t, \boldsymbol{x}}\left[\boldsymbol{X}_{t+\tau}^{V}\right]^{(r)}-\mathbb{E}^{t, \boldsymbol{x}}\left[\boldsymbol{Z}_{t+\tau}^{V}\right]^{(r)}\right|=\mathcal{O}\left(V^{-3 \beta}\right) .
\end{aligned}
$$

Proof. It is straightforward to prove (3.23) by (3.15) and (3.18). To prove (3.24), using Lemma 3.4 and Lemma 3.6 we obtain

$$
\begin{aligned}
\mid \mathbb{E}^{t, \boldsymbol{x}}\left[\boldsymbol{X}_{t+\tau}^{V}\right]^{(r)}-\mathbb{E}^{t, \boldsymbol{x}}\left[\boldsymbol{Z}_{t+\tau}^{V}\right]^{(r) \mid=} & \frac{\tau^{2}}{2}\left(a_{j}^{V, k}-\hat{a}_{j}^{V, k}\right) a_{k}^{V}\left[\boldsymbol{x}^{(r)}\right]^{V, j}+\frac{\tau^{2}}{2} V^{-1} a_{j}^{V, k} a_{k}^{V}\left[\boldsymbol{x}^{(r)}\right]^{V, j k} \\
& +\frac{\tau^{3}}{2} a_{j}^{V}\left(a_{k}^{V, l}-\hat{a}_{k}^{V, l}\right) a_{l}^{V}\left[\boldsymbol{x}^{(r)}\right]^{V, j k} \\
& +\frac{\tau^{3}}{6} a_{j}^{V, k} a_{k}^{V, l} a_{l}^{V}\left[\boldsymbol{x}^{(r)}\right]^{V, j}+\frac{\tau^{3}}{6} a_{j}^{V, k l} a_{k}^{V} a_{l}^{V}\left[\boldsymbol{x}^{(r)}\right]^{V, j} \\
& -\frac{\tau^{3}}{8} \hat{a}_{j}^{V, k l} a_{k}^{V} a_{l}^{V}\left[\boldsymbol{x}^{(r)}\right]^{V, j}+\mathcal{O}\left(V^{-4 \beta}\right) .
\end{aligned}
$$

Using the scaling relation (3.7), the assumption $\beta<1$, and the fact

$$
a_{j}^{V, k}-\hat{a}_{j}^{V, k}=\mathcal{O}\left(V^{-1}\right),
$$

we get the order of the leading terms is $\mathcal{O}\left(V^{-3 \beta}\right)$.

The result is consistent with that in [1]. Here special attention should be paid to the term

$$
\frac{\tau^{2}}{2} V^{-1} a_{j}^{V, k} a_{k}^{V}\left[\boldsymbol{x}^{(r)}\right]^{V, j k} .
$$

in (3.25). It is negligible compared with $\mathcal{O}\left(\tau^{3}\right)$ in large volume scaling. However, as we will reveal in the following subsection, the analogous term in the covariance will become dominant.

\subsection{Local truncation errors for the covariance.}

Proposition 3.8. If $\boldsymbol{X}_{t}$ satisfies Equation (1.3), then for given $0<\beta<1, \operatorname{Cov}\left(\boldsymbol{X}_{t+\tau}^{V}\right)$ has the following expansion:

$$
\begin{aligned}
\operatorname{Cov}\left(\boldsymbol{X}_{t+\tau}^{V}\right)= & \tau V^{-1} a_{j}^{V} \boldsymbol{\nu}_{j}^{(2)}+\frac{\tau^{2}}{2} V^{-1} a_{j}^{V, k} a_{k}^{V} \boldsymbol{\nu}_{j}^{(2)} \\
& +\frac{\tau^{2}}{2} V^{-1} a_{j}^{V, k} a_{k}^{V}\left(\boldsymbol{\nu}_{j} \boldsymbol{\nu}_{k}+\boldsymbol{\nu}_{k} \boldsymbol{\nu}_{j}\right)+\mathcal{O}\left(V^{-3 \beta-1}\right) .
\end{aligned}
$$

Proof. Given $0<\beta<1$, there exists a positive integer $\zeta$ such that $\zeta \beta>3 \beta+1$. Then we apply Theorem 2.14 to $\boldsymbol{X}_{t}$ until $\zeta$-th order and transfer to the rescaled variables. Notice that

$$
a_{j}^{\mathbb{I}(j))} \rightarrow V^{1-\#(\mathbb{I}(j))} a_{j}^{V, \mathbb{I}(j)} .
$$


So the power of $V$ for the rescaled coefficients is

$$
\sum_{j=1}^{q}\left(1-\#\left(\mathbb{I}\left(i_{j}\right)\right)\right)-2=-\lambda_{q} \leq-1 .
$$

Therefore

$$
\begin{aligned}
\operatorname{Cov}\left(\boldsymbol{X}_{t+\tau}^{V}\right)= & \tau V^{-1} a_{j}^{V} \boldsymbol{\nu}_{j}^{(2)}+\frac{\tau^{2}}{2} V^{-1} a_{j}^{V, k} a_{k}^{V} \boldsymbol{\nu}_{j}^{(2)} \\
& +\frac{\tau^{2}}{2} V^{-1} a_{j}^{V, k} a_{k}^{V}\left(\boldsymbol{\nu}_{j} \boldsymbol{\nu}_{k}+\boldsymbol{\nu}_{k} \boldsymbol{\nu}_{j}\right)+\mathcal{O}\left(V^{-3 \beta-1}\right)+\mathcal{O}\left(V^{-\zeta \beta}\right) \\
= & \tau V^{-1} a_{j}^{V} \boldsymbol{\nu}_{j}^{(2)}+\frac{\tau^{2}}{2} V^{-1} a_{j}^{V, k} a_{k}^{V} \boldsymbol{\nu}_{j}^{(2)} \\
& +\frac{\tau^{2}}{2} V^{-1} a_{j}^{V, k} a_{k}^{V}\left(\boldsymbol{\nu}_{j} \boldsymbol{\nu}_{k}+\boldsymbol{\nu}_{k} \boldsymbol{\nu}_{j}\right)+\mathcal{O}\left(V^{-3 \beta-1}\right)
\end{aligned}
$$

The proof is completed.

We have the following estimates for the covariance of $\boldsymbol{Y}_{t}^{V}$ and $\boldsymbol{Z}_{t}^{V}$.

Proposition 3.9. If $\boldsymbol{Y}_{t}, \boldsymbol{Z}_{t}$ satisfy Equation (3.8), (3.10) respectively, then

$$
\begin{aligned}
& \operatorname{Cov}\left(\boldsymbol{Y}_{t+\tau}^{V}\right)=\tau V^{-1} a_{j}^{V} \boldsymbol{\nu}_{j}^{(2)}+\mathcal{O}\left(V^{-3 \beta-1}\right), \\
& \operatorname{Cov}\left(\boldsymbol{Z}_{t+\tau}^{V}\right)=\tau V^{-1} a_{j}^{V} \boldsymbol{\nu}_{j}^{(2)}+\frac{\tau^{2}}{2} V^{-1} \hat{a}_{j}^{V, k} a_{k}^{V} \boldsymbol{\nu}_{j}^{(2)}+\mathcal{O}\left(V^{-3 \beta-1}\right) .
\end{aligned}
$$

Proof. Equation (3.29) is a direct application of (3.26) with the condition that the propensity functions $a_{k}$ are constants. To prove (3.30), we have from (3.21)

$$
\begin{aligned}
\operatorname{Cov}\left(\boldsymbol{Z}_{t+\tau}^{V}\right)= & \boldsymbol{x}^{(2)}+\tau a_{j}^{V}\left(\boldsymbol{\mu}^{V}(\boldsymbol{x})\right)\left[\boldsymbol{x}^{(2)}\right]^{V, j}+\frac{\tau^{2}}{2} a_{j}^{V}\left(\boldsymbol{\mu}^{V}(\boldsymbol{x})\right) a_{k}^{V}\left(\boldsymbol{\mu}^{V}(\boldsymbol{x})\right)\left[\boldsymbol{x}^{(2)}\right]^{V, j k} \\
& -\left[\boldsymbol{x}+\tau a_{j}^{V}\left(\boldsymbol{\mu}^{V}(\boldsymbol{x})\right) \boldsymbol{x}^{V, j}\right]^{(2)}
\end{aligned}
$$

From the expansion

$$
a_{j}^{V}\left(\boldsymbol{\mu}^{V}(\boldsymbol{x})\right)=a_{j}^{V}(\boldsymbol{x})+\frac{1}{2} \tau \hat{a}_{j}^{V, k} a_{k}^{V}+\mathcal{O}\left(V^{-2 \beta}\right),
$$

we obtain

$$
\begin{aligned}
\operatorname{Cov}\left(\boldsymbol{Z}_{t+\tau}^{V}\right) & =\tau a_{j}^{V}\left(\boldsymbol{\mu}^{V}(\boldsymbol{x})\right)\left(\left[\boldsymbol{x}^{(2)}\right]^{V, j}-\boldsymbol{x} \boldsymbol{x}^{V, j}-\boldsymbol{x}^{V, j} \boldsymbol{x}\right) \\
& =\tau V^{-1} a_{j}^{V} \boldsymbol{\nu}_{j}^{(2)}+\frac{\tau^{2}}{2} V^{-1} \hat{a}_{j}^{V, k} a_{k}^{V} \boldsymbol{\nu}_{j}^{(2)}+\mathcal{O}\left(V^{-3 \beta-1}\right) .
\end{aligned}
$$

The proof is completed.

Now we have the covariance error of Euler tau-leaping and midpoint tau-leaping.

Theorem 3.10 (LTE for the covariance). If $\boldsymbol{X}_{t}, \boldsymbol{Y}_{t}$, and $\boldsymbol{Z}_{t}$ satisfy Equations (1.3), (3.8), and (3.10) respectively, then

$$
\begin{aligned}
\left|\operatorname{Cov}\left(\boldsymbol{X}_{t+\tau}^{V}\right)-\operatorname{Cov}\left(\boldsymbol{Y}_{t+\tau}^{V}\right)\right| & =\mathcal{O}\left(V^{-2 \beta-1}\right), \\
\left|\operatorname{Cov}\left(\boldsymbol{X}_{t+\tau}^{V}\right)-\operatorname{Cov}\left(\boldsymbol{Z}_{t+\tau}^{V}\right)\right| & =\mathcal{O}\left(V^{-2 \beta-1}\right) .
\end{aligned}
$$


Proof. Thanks to Propositions 3.8 and 3.9, we have

$$
\begin{aligned}
\operatorname{Cov}\left(\boldsymbol{X}_{t+\tau}^{V}\right)-\operatorname{Cov}\left(\boldsymbol{Y}_{t+\tau}^{V}\right)= & \frac{\tau^{2}}{2} V^{-1} a_{j}^{V, k} a_{k}^{V}\left(\boldsymbol{\nu}_{j} \boldsymbol{\nu}_{k}+\boldsymbol{\nu}_{k} \boldsymbol{\nu}_{j}\right)+\frac{\tau^{2}}{2} V^{-1} a_{j}^{V, k} a_{k}^{V} \boldsymbol{\nu}_{j}^{(2)} \\
& +\mathcal{O}\left(V^{-3 \beta-1}\right), \\
\operatorname{Cov}\left(\boldsymbol{X}_{t+\tau}^{V}\right)-\operatorname{Cov}\left(\boldsymbol{Z}_{t+\tau}^{V}\right)= & \frac{\tau^{2}}{2} V^{-1} a_{j}^{V, k} a_{k}^{V}\left(\boldsymbol{\nu}_{j} \boldsymbol{\nu}_{k}+\boldsymbol{\nu}_{k} \boldsymbol{\nu}_{j}\right) \\
& +\frac{\tau^{2}}{2} V^{-1}\left(a_{j}^{V, k}-\hat{a}_{j}^{V, k}\right) a_{k}^{V} \boldsymbol{\nu}_{j}^{(2)}+\mathcal{O}\left(V^{-3 \beta-1}\right) .
\end{aligned}
$$

Noticing that $a_{j}^{V, k}-\hat{a}_{j}^{V, k} \sim \mathcal{O}\left(V^{-1}\right)$, we get the desired result.

The above result shows that the covariance obtained by midpoint tau-leaping is no more accurate than that of tau-leaping, at least in order. This is very interesting because though both methods achieve high order in the large volume scaling, the local consistency orders are the same $-\mathcal{O}\left(\tau^{2} V^{-1}\right)$ ! This fact is in sharp contrast with the result for approximating the moments about the origin, in which midpoint tau-leaping is shown to be superior to Euler tau-leaping. This paradoxical analysis of the order originates from the essential difference between the infinitesimal generators for deterministic ODEs and jump process here.

Theorem 3.10 is also instructive for explaining the numerical behavior of Euler tau-leaping and midpoint tau-leaping for computing the covariance in the non-scaled version in Section 5. We will observe that numerically both methods performs similarly as first order schemes. We explain this numerical behavior from the fact that the system size is kept invariant in realistic computations. A careful observation reveals that the additional order in Equations (3.32) and (3.33) comes from the term $\mathcal{O}\left(V^{-1}\right)$, which is due to the rescaling of the system size. After abandoning this effect we obtain the leading truncation terms with $\mathcal{O}\left(\tau^{2}\right)$, which suggests global first order accuracy. This is consistent with the practical computations. Based on the above considerations we claim that the realistic numerical order for any leaping scheme can be analyzed in the following 3 steps.

- Step 1. Write down the local truncation error estimate in the traditional scaling $\tau \rightarrow 0$;

- Step 2. Reorder the error terms according to the magnitude of the power of $V$ under the large volume scaling;

- Step 3. Abandon the dependence on $V$ in the leading order terms and find the lowest order $p$ on $\tau$, which is the weak local order.

REMARK 3.3. The special consideration for the truncation error analysis of the covariance also highlights the necessity of design for the higher order methods in tau-leaping simulations, which is one aim of our future work.

\section{Global weak convergence in the large volume scaling}

This section is concerned with the global weak convergence of general explicit tau-leaping type methods in large volume scaling. It establishes the weak convergence order of a tau-leaping method resting on properties of its one-step approximation only. This work is motivated by Anderson et al in [1], where weak convergence for Euler tau-leaping and mid-point tau-leaping in large volume scaling are proved. 
4.1. Assumptions and notations. Consider a general explicit tau-leaping type approximation

$$
\overline{\boldsymbol{X}}_{t+\tau}=\overline{\boldsymbol{X}}_{t}+A\left(\overline{\boldsymbol{X}}_{t}, \tau ; \boldsymbol{\xi}_{0}, \ldots, \boldsymbol{\xi}_{s}\right)
$$

where $\boldsymbol{\xi}_{u}(u=0,1, \ldots, s)$ are random vectors depending on $\overline{\boldsymbol{X}}_{t}, \tau, \boldsymbol{\xi}_{0}, \ldots, \boldsymbol{\xi}_{u-1}$, and $A$ is a vector function with integer values. It is easy to see that Euler tau-leaping, midpoint tau-leaping and Poisson Runge-Kutta tau-leaping are among this framework. For instance, Euler tau-leaping can be expressed as

$$
A^{E}\left(\overline{\boldsymbol{X}}_{t}, \tau ; \boldsymbol{\xi}_{0}\right)=\sum_{j=1}^{M} \boldsymbol{\nu}_{j} \xi_{0, j},
$$

where $\boldsymbol{\xi}_{0}=\left\{\xi_{0, j}\right\}_{j=1}^{M}=\left\{\mathcal{P}_{j}\left(a_{j}\left(\overline{\boldsymbol{X}}_{t}\right) \tau\right)\right\}_{j=1}^{M}$.

Without loss of generality, we suppose the interval $[0, T]$ is partitioned into $n$ subintervals with stepsize $\tau=T / n$, that is, $0=t_{0}<t_{1}<\cdots<t_{n}=T$ and $t_{k+1}-t_{k}=\tau$. According to (4.1), we construct the numerical solutions

$$
\overline{\boldsymbol{X}}_{0}=\boldsymbol{X}_{0}, \overline{\boldsymbol{X}}_{k+1}=\overline{\boldsymbol{X}}_{k}+A\left(\overline{\boldsymbol{X}}_{k}, \tau ; \boldsymbol{\xi}_{0, k}, \ldots, \boldsymbol{\xi}_{s, k}\right), k=0,1, \ldots, n-1 .
$$

In the large volume scaling, the approximation (4.1) becomes

$$
\overline{\boldsymbol{X}}_{t+\tau}^{V}=\boldsymbol{x}+A^{V}\left(\boldsymbol{x}, \tau ; \boldsymbol{\xi}_{0}^{V}, \ldots, \boldsymbol{\xi}_{s}^{V}\right) .
$$

provided $\boldsymbol{x}=\overline{\boldsymbol{X}}_{t} / V$ as before. For example, Euler tau-leaping has the form

$$
\left(A^{E}\right)^{V}\left(\boldsymbol{x}, \tau ; \boldsymbol{\xi}_{0}\right)=\sum_{j=1}^{M} \frac{\boldsymbol{\nu}_{j}}{V} \xi_{0, j}^{V},
$$

where $\boldsymbol{\xi}_{0}^{V}=\left\{\xi_{0, j}^{V}\right\}_{j=1}^{M}=\left\{\mathcal{P}_{j}\left(V a_{j}^{V}(\boldsymbol{x}) \tau\right)\right\}_{j=1}^{M}$. Thus we have the transformed numerical schemes

$$
\overline{\boldsymbol{X}}_{0}^{V}=\boldsymbol{X}_{0} / V, \overline{\boldsymbol{X}}_{k+1}^{V}=\overline{\boldsymbol{X}}_{k}^{V}+A^{V}\left(\overline{\boldsymbol{X}}_{k}^{V}, \tau ; \boldsymbol{\xi}_{0, k}^{V}, \ldots, \boldsymbol{\xi}_{s, k}^{V}\right), k=0,1, \ldots, n-1 .
$$

To formulate the general convergence theorem, we borrow some notations from [1]. Let $\mathbb{L}^{V}=\left\{\boldsymbol{y}: \boldsymbol{y}=\boldsymbol{X} / V, \boldsymbol{X} \in \mathbb{Z}^{N}\right\}$. For $\boldsymbol{x} \in \mathbb{L}^{V}$ and any function $f(\boldsymbol{x}) \in C_{0}^{\infty}\left(\mathbb{R}^{N}\right)$, we define the Markov semigroup

$$
\left(\mathscr{T}_{t} f\right)(\boldsymbol{x})=v(t, \boldsymbol{x}):=\mathbb{E}^{0, \boldsymbol{x}} f\left(\boldsymbol{X}_{t}^{V}\right),
$$

and the Markov semigroup $T_{\tau}$ associated with the numerical method (4.3) by

$$
\left(T_{\tau} f\right)(\boldsymbol{x}):=\mathbb{E}^{0, \boldsymbol{x}} f\left(\overline{\boldsymbol{X}}_{1}^{V}\right),
$$

with initial condition $\boldsymbol{X}_{0}^{V}=\boldsymbol{x}$ for $\boldsymbol{X}_{t}^{V}$. Standard results give that $v(t, \boldsymbol{x})$ satisfies the following backward equation

$$
\begin{aligned}
\frac{\partial v(t, \boldsymbol{x})}{\partial t} & =\mathcal{L}^{V} v(t, \boldsymbol{x})=a_{j}^{V} v^{V, j}(t, \boldsymbol{x})=V a_{j}^{V}(\boldsymbol{x})\left(v\left(t, \boldsymbol{x}+\boldsymbol{\nu}_{j} / V\right)-v(t, \boldsymbol{x})\right), \\
v(0, \boldsymbol{x}) & =f(\boldsymbol{x}), \boldsymbol{x} \in \mathbb{L}^{V},
\end{aligned}
$$


where the Einstein summation convention is applied. The above equation can be viewed as a finite dimensional linear system because of Condition 3 in Assumption 3.1 on the intensity functions $a_{j}^{V}$. That is, recalling that $a_{j}^{V}(\boldsymbol{x})=0$ for all $\boldsymbol{x}$ outside the compact set $\Omega$, we have $v(t, \boldsymbol{x})=f(\boldsymbol{x})$ for all $t>0$ and $\boldsymbol{x} \notin \Omega$.

Definition 4.1 (The function space $D_{0}^{Q}\left(\mathbb{R}^{N}\right)$ ). We define the function space $D_{0}^{Q}\left(\mathbb{R}^{N}\right)$ as the set of functions $f(\boldsymbol{x})$ which have compact support in $\mathbb{R}^{N}$ and satisfy the condition

$$
\sup _{i=0,1, \ldots, Q} \sup _{j_{1}, \ldots, j_{i} \leq M} \sup _{\boldsymbol{x} \in \mathbb{L}^{V}}\left|f^{V, j_{1} j_{2} \ldots j_{i}}(\boldsymbol{x})\right| \leq K_{Q},
$$

where the positive constant $K_{Q}$ is independent of $V$.

Definition 4.2 (Weak $p$-th order consistency). We say that a tau-leaping type scheme has weak $p$-th order consistency if for any $\boldsymbol{x} \in \mathbb{L}^{V}$ and $f(\boldsymbol{x}) \in D_{0}^{p+1}\left(\mathbb{R}^{N}\right)$, there exists a constant $K>0$, independent of $V$ and $\boldsymbol{x}$, such that

$$
\left|\left(\mathscr{T}_{\tau} f\right)(\boldsymbol{x})-\left(T_{\tau} f\right)(\boldsymbol{x})\right| \leq K \sup _{i=0,1, \ldots, p+1} \sup _{j_{1}, \ldots, j_{i} \leq M} \sup _{\boldsymbol{x} \in \mathbb{L}^{V}}\left|f^{V, j_{1} j_{2} \ldots j_{i}}(\boldsymbol{x})\right| \tau^{p+1}
$$

for sufficiently small $\tau=V^{-\beta}$ as $V \rightarrow \infty$.

4.2. Global weak convergence. We will establish a general global weak convergence theorem for the explicit tau-leaping type method based on its one-step local truncation error in the large volume scaling. This is a generalization of the work by Anderson et al in [1], where the weak convergence for Euler tau-leaping and mid-point tau-leaping in large volume scaling is proved. However we take the classical approach for proving the convergence of numerical ODEs, which says that $\mathcal{O}\left(\tau^{p+1}\right)$ local error implies $\mathcal{O}\left(\tau^{p}\right)$ global error since the stability condition is satisfied automatically for explicit one-step methods.

To prove global weak convergence, we need the following lemma for the regularity of higher order differences of $v(t, \boldsymbol{x})$ compared with those in [1].

Lemma 4.3 (Regularity of $v(t, \boldsymbol{x})$ ). Suppose the initial data $f(\boldsymbol{x}) \in C_{0}^{p+1}\left(\mathbb{R}^{N}\right)$. Then there exists constants $K_{i}$ independent of $V$ such that

$$
\sup _{j_{1}, \ldots, j_{i} \leq M} \sup _{t \leq T} \sup _{\boldsymbol{x} \in \mathbb{L}^{V}}\left|v^{V, j_{1} \ldots j_{i}}(t, \boldsymbol{x})\right| \leq K_{i}, i=0,1, \ldots, p+1 .
$$

The proof of this Lemma will be deferred to the end of this subsection. Now we present our global weak convergence theorem.

TheOREM 4.4. Under the Assumption 3.1 and the weak p-th order consistency condition (Definition 4.2), we have the following global weak $p$-th order convergence:

$$
\left|\left(\mathscr{T}_{n \tau} f\right)(\boldsymbol{x})-\left(T_{\tau}^{n} f\right)(\boldsymbol{x})\right| \leq C \tau^{p}, n \tau \leq T
$$

for sufficient small $\tau=V^{-\beta}$ as $V \rightarrow \infty$, where $C$ is a constant independent of $V$.

REMARK 4.1. The readers may easily deduce the local truncation error estimates for the explicit Euler tau-leaping and midpoint tau-leaping methods based on the analysis in Sections 2 and 3. This will lead to the same weak convergence results in [1]. We will omit the details here. But this framework does not in principle contain the global weak convergence analysis for the moments in Section 3 since $\boldsymbol{x}^{(r)}$ is not 
compactly supported. It also does not contain the analysis for the covariance, even for the compactly supported $f(\boldsymbol{x})$. because the covariance function $\operatorname{Cov}(f(\boldsymbol{x}))$ cannot be represented as the expectation of a single function $g(\boldsymbol{x})$.

Proof. (of Theorem 4.4)

$$
\begin{aligned}
\left|\left(\mathscr{T}_{n \tau} f\right)(\boldsymbol{x})-\left(T_{\tau}^{n} f\right)(\boldsymbol{x})\right| & =\left|\sum_{k=1}^{n}\left(T_{\tau}^{k-1}\left(\mathscr{T}_{\tau}-T_{\tau}\right) \mathscr{T}_{\tau(n-k)} f\right)(\boldsymbol{x})\right| \\
& \leq \sum_{k=1}^{n} \mathbb{E}^{0, \boldsymbol{x}}\left|\left(\mathscr{T}_{\tau}-T_{\tau}\right)\left(\mathscr{T}_{\tau(n-k)} f\right)\left(\overline{\boldsymbol{X}}_{k-1}^{V}\right)\right|
\end{aligned}
$$

Notice that $\left(\mathscr{T}_{\tau(n-k)} f\right)(\boldsymbol{x})=v(\tau(n-k), \boldsymbol{x}) \in D_{0}^{p+1}\left(\mathbb{R}^{N}\right)$ for $k=0,1, \ldots, n$ from Lemma 4.3. Combining Definition 4.2 and Lemma 4.3 we have

$$
\begin{aligned}
\left|\left(\mathscr{T}_{n \tau} f\right)(\boldsymbol{x})-\left(T_{\tau}^{n} f\right)(\boldsymbol{x})\right| & \leq \sum_{k=1}^{n} \mathbb{E}^{0, \boldsymbol{x}}\left|\left(\mathscr{T}_{\tau}-T_{\tau}\right) v\left(\tau(n-k), \overline{\boldsymbol{X}}_{k-1}^{V}\right)\right| \\
& \leq K \sum_{k=1}^{n} \sup _{i=0,1, \ldots, p+1} \sup _{j_{1}, \ldots, j_{i} \leq M} \sup _{\boldsymbol{x} \in \mathbb{L}^{V}}\left|v^{V, j_{1} j_{2} \ldots j_{i}}(\tau(n-k), \boldsymbol{x})\right| \tau^{p+1} \\
& \leq K T \tau^{p} \sup _{i=0,1, \ldots, p+1} \sup _{j_{1}, \ldots, j_{i} \leq M} \sup _{t \leq T} \sup _{\boldsymbol{x} \in \mathbb{L}^{V}}\left|v^{V, j_{1} j_{2} \ldots j_{i}}(t, \boldsymbol{x})\right|, \\
& \leq C \tau^{p} .
\end{aligned}
$$

which is the desired result.

Proof. (of Lemma 4.3) We build the regularity estimate (4.9) by induction. The cases $i=1,2$ have been established in [1]. The case $i=0$ can also be established similarly, and is omitted here. In fact, to prove that the estimate holds for bigger $i$ we will find the proof of essentially the estimate of the leading order is always similar as proving it for smaller $i$ cases because of the special "convective"-type structure of the system which $v$ satisfies.

Let us define $\boldsymbol{I}=\left\{j_{1}, j_{2}, \ldots, j_{i}\right\}$ and $\widehat{\boldsymbol{I}}_{k}=\left\{j_{1}, \ldots, j_{k-1}, j_{k+1}, \ldots, j_{i}\right\}, k=1,2, \ldots, i$. We proceed with the two steps described below. The Einstein summation convention is not applied here.

Step 1: First establish the differential equation which $v^{V, \boldsymbol{I}}$ satisfies.

$$
\begin{aligned}
\partial_{t} v^{V, \boldsymbol{I}}(t, \boldsymbol{x})= & V\left(-a_{0}^{V}(\boldsymbol{x}) v^{V, \boldsymbol{I}}(t, \boldsymbol{x})+\sum_{j=1}^{M} a_{j}^{V}(\boldsymbol{x}) v^{V, \boldsymbol{I}}\left(t, \boldsymbol{x}+\boldsymbol{\nu}_{j} / V\right)\right) \\
& +\sum_{k=1}^{i} \sum_{j=1}^{M} a_{j}^{V, j_{k}} v^{V, \widehat{\boldsymbol{I}}_{k}, j}\left(t, \boldsymbol{x}+\boldsymbol{\nu}_{j_{k}} / V\right)+w(t, \boldsymbol{x} ; \boldsymbol{I}),
\end{aligned}
$$

where $a_{0}^{V}(\boldsymbol{x})$ is the rescaled total propensity function, $w(t, \boldsymbol{x} ; \boldsymbol{I})$ only involves the summation of finite lower order difference terms including the product of some $a_{j}^{V, \tilde{\boldsymbol{I}}_{1}} \mathrm{~s}$ and one $v^{V, \tilde{\boldsymbol{I}}_{2}}(t, \boldsymbol{y})\left(\boldsymbol{y} \in \mathbb{L}^{V}\right)$, where both $\tilde{\boldsymbol{I}}_{1}$ and $\tilde{\boldsymbol{I}}_{2}$ are index sets, $\#\left(\tilde{\boldsymbol{I}}_{1}\right) \leq i$ but $\#\left(\tilde{\boldsymbol{I}}_{2}\right)<i$.

The proof of (4.11) can be completed by induction. At first it is obvious when $i=0,1$. Suppose it holds for $\boldsymbol{I}=\left\{j_{1}, \ldots, j_{i}\right\}$, and take the difference operation with respect to $j_{i+1}$ to both sides of (4.11). With the fact

$$
(g h)^{V, j}(\boldsymbol{x})=g(\boldsymbol{x}) h^{V, j}(\boldsymbol{x})+g^{V, j}(\boldsymbol{x}) h\left(\boldsymbol{x}+\boldsymbol{\nu}_{j} / V\right),
$$


we have

$$
\begin{aligned}
\partial_{t} v^{V, \boldsymbol{I}, j_{i+1}}(t, \boldsymbol{x})= & V\left(-a_{0}^{V}(\boldsymbol{x}) v^{V, \boldsymbol{I}, j_{i+1}}(t, \boldsymbol{x})+\sum_{j=1}^{M} a_{j}^{V}(\boldsymbol{x}) v^{V, \boldsymbol{I}, j_{i+1}}\left(t, \boldsymbol{x}+\boldsymbol{\nu}_{j} / V\right)\right) \\
& +V\left(-a_{0}^{V, j_{i+1}}(\boldsymbol{x}) v^{V, \boldsymbol{I}}\left(t, \boldsymbol{x}+\boldsymbol{\nu}_{j_{i+1}} / V\right)\right. \\
& \left.+\sum_{j=1}^{M} a_{j}^{V, j_{i+1}}(\boldsymbol{x}) v^{V, \boldsymbol{I}}\left(t, \boldsymbol{x}+\boldsymbol{\nu}_{j} / V+\boldsymbol{\nu}_{j_{i+1}} / V\right)\right) \\
& +\sum_{k=1}^{i} \sum_{j=1}^{M} a_{j}^{V, j_{k}}(\boldsymbol{x}) v^{V, \widehat{\boldsymbol{I}}_{k}, j, j_{i+1}}\left(t, \boldsymbol{x}+\boldsymbol{\nu}_{j_{k}} / V\right)+w\left(t, \boldsymbol{x} ; \boldsymbol{I}, j_{i+1}\right) \\
= & P_{1}+P_{2}+P_{3},
\end{aligned}
$$

where $P_{1}, P_{2}$, and $P_{3}$ correspond to the different lines above. We have

$$
P_{2}=\sum_{j=1}^{M} a_{j}^{V, j_{i+1}}(\boldsymbol{x}) v^{V, \boldsymbol{I}, j}\left(t, \boldsymbol{x}+\boldsymbol{\nu}_{j_{i+1}} / V\right) .
$$

Combining this with $P_{3}$ we obtain that Equation (4.11) also holds for the $i+1$ case. The induction is finished.

Step 2: Prove the regularity estimate (4.9) by induction.

Suppose it holds for $i-1$ case. Notice that (4.11) can be viewed as a finite dimensional ODE system with the label $(\boldsymbol{I}, \boldsymbol{x})$ as its one component, and define the $L^{\infty}$-norm of $v$ with respect to all of the possible labels $(\boldsymbol{I}, \boldsymbol{x})$ as

$$
\left\|v^{V, \boldsymbol{I}}(t, \boldsymbol{x})\right\|_{\infty}:=\sup _{j_{1}, \ldots, j_{i} \leq M} \sup _{\boldsymbol{x} \in \mathbb{L}^{V}}\left|v^{V, j_{1} \ldots j_{i}}(t, \boldsymbol{x})\right| .
$$

Suppose in time $\left[0, T_{1}\right]$ this $L^{\infty}$-norm is achieved at the label $(\boldsymbol{I}, \boldsymbol{x})$. We have from $(4.11)$

$$
\begin{aligned}
\frac{1}{2} \partial_{t}\left(v^{V, \boldsymbol{I}}(t, \boldsymbol{x})\right)^{2}= & -V a_{0}^{V}(\boldsymbol{x})\left(v^{V, \boldsymbol{I}}(t, \boldsymbol{x})\right)^{2}+V \sum_{j=1}^{M} a_{j}^{V}(\boldsymbol{x}) v^{V, \boldsymbol{I}}\left(t, \boldsymbol{x}+\boldsymbol{\nu}_{j} / V\right) v^{V, \boldsymbol{I}}(t, \boldsymbol{x}) \\
& +\sum_{k=1}^{i} \sum_{j=1}^{M} a_{j}^{V, j_{k}} v^{V, \widehat{\boldsymbol{I}}_{k}, j}\left(t, \boldsymbol{x}+\boldsymbol{\nu}_{j_{k}} / V\right) v^{V, \boldsymbol{I}}(t, \boldsymbol{x})+w(t, \boldsymbol{x} ; \boldsymbol{I}) v^{V, \boldsymbol{I}}(t, \boldsymbol{x}) \\
\leq & -V a_{0}^{V}(\boldsymbol{x})\left(v^{V, \boldsymbol{I}}(t, \boldsymbol{x})\right)^{2}+V a_{0}^{V}(\boldsymbol{x})\left\|v^{V, \boldsymbol{I}}(t, \boldsymbol{x})\right\|_{\infty}^{2} \\
& +\left(i M \sup _{\boldsymbol{x} \in \mathbb{L}^{V}} \sup _{j, k \leq M}\left|a_{j}^{V, k}(\boldsymbol{x})\right|+\frac{1}{2}\right)\left\|v^{V, \boldsymbol{I}}(t, \boldsymbol{x})\right\|_{\infty}^{2}+\frac{1}{2}|w(t, \boldsymbol{x} ; \boldsymbol{I})|^{2} \\
\leq & K_{i}\left\|v^{V, \boldsymbol{I}}(t, \boldsymbol{x})\right\|_{\infty}^{2}+K_{\boldsymbol{I}},
\end{aligned}
$$

where

$$
K_{i}=i M \sup _{\boldsymbol{x} \in \mathbb{L}^{V}} \sup _{j, k \leq M}\left|a_{j}^{V, k}(\boldsymbol{x})\right|+\frac{1}{2}, K_{\boldsymbol{I}}=\frac{1}{2} \sup _{t \leq T} \sup _{\boldsymbol{x} \in \mathbb{L}^{V}}|w(t, \boldsymbol{x} ; \boldsymbol{I})|^{2}
$$

are positive constants independent of $\boldsymbol{x}, t$, and $V$ from the Assumption 3.1 and the induction hypothesis. Thus we obtain

$$
\left\|v^{V, \boldsymbol{I}}(t, \boldsymbol{x})\right\|_{\infty}^{2} \leq\left\|f^{V, \boldsymbol{I}}(\boldsymbol{x})\right\|_{\infty}^{2}+2 K_{i} \int_{0}^{t}\left\|v^{V, \boldsymbol{I}}(s, \boldsymbol{x})\right\|_{\infty}^{2} d s+K_{\boldsymbol{I}} t
$$




\begin{tabular}{|c|c|c|c|}
\hline$\tau$ & tauleap & midpoint & GRC \\
\hline \hline 0.8 & $-127.65(1.87)$ & $-3.44(3.77)$ & $-3.45(3.77)$ \\
\hline 0.4 & $-66.15(1.93)$ & $-0.89(3.85)$ & $-0.89(3.89)$ \\
\hline 0.2 & $-33.69(1.96)$ & $-0.22(3.99)$ & $-0.22(4.01)$ \\
\hline 0.1 & $-17.00(1.98)$ & $-0.06(3.59)$ & $-0.06(4.02)$ \\
\hline
\end{tabular}

TABLE 5.1. Error of the mean for the Euler tau-leaping method (tauleap), the midpoint tauleaping method (midpoint), and the GRC-tau-leaping method (GRC). The first column shows the time stepsize. The quantities outside of the parentheses from column 2 to 4 are the numerical errors with the corresponding $\tau$. The constants in the parentheses from column 2 to 4 show the ratio of the error in the upper row over the error in the current row (we do not show the results with $\tau=1.6$ ). The result implies that only Euler tau-leaping method is of first order, the other two methods are of second order for the mean.

for $t \in\left[0, T_{1}\right]$. From Gronwall's inequality we have

$$
\left\|v^{V, \boldsymbol{I}}(t, \boldsymbol{x})\right\|_{\infty}^{2} \leq\left(\left\|f^{V, \boldsymbol{I}}(\boldsymbol{x})\right\|_{\infty}^{2}++K_{\boldsymbol{I}} T\right) \exp \left(2 K_{i} T\right) .
$$

where $\left\|f^{V, \boldsymbol{I}}(\boldsymbol{x})\right\|_{\infty}^{2}$ is bounded since $f \in C_{0}^{p+1}\left(\mathbb{R}^{N}\right)$. We can continue this process in time $\left[T_{1}, T_{2}\right],\left[T_{2}, T_{3}\right], \ldots$ until the final time $T$ is achieved. Thus the induction is finished.

\section{Numerical comparisons}

In this section we numerically check the aforementioned analysis on different methods. We compare the Euler tau-leaping method, the midpoint tau-leaping method, and the Gaussian random correction tau-leaping method (GRC-tau-leaping) [22]. We first summarize some theoretical results.

- The Euler tau-leaping method. The Euler tau-leaping method is first order consistent for all moments and the covariance in both traditional and large volume scaling.

- The midpoint tau-leaping method (unrounded version). The midpoint tau-leaping method is second order consistent for all moments and first order consistent for the covariance in the large volume scaling.

- The Gaussian random correction tau-leaping method (GRC-tauleaping). The GRC-tau-leaping method is second order consistent for both the mean and covariance in the traditional scaling. We will not list the scheme here. The readers are referred to [22] for more details.

Here $p$-th order consistency means that the leading remainder term in LTE is $\mathcal{O}\left(\tau^{p+1}\right)$.

Next we implement the above mentioned methods to a simple model problem ${ }^{1}$

$$
S \rightarrow 2 S \text {. }
$$

This system has one reaction with propensity function $a(x)=c x$, where the rate constant is $c=0.1$. The state-change vector is $\nu=1$. The initial condition is $X_{0}=$ 1000. The exact mean and variance of $X_{T}$ can be solved explicitly, $\mathbb{E}\left[X_{T}\right]=X_{0} e^{c T}$,

\footnotetext{
${ }^{1}$ We did not choose the more commonly used example $S \rightarrow \emptyset$ because in it the number of molecules of $S$ decays exponentially with time $T$ and the numerical errors will be comparable with sampling fluctuations, which prevent us from observing clear order relations.
} 


\begin{tabular}{|c|c|c|c|}
\hline$\tau$ & tauleap & midpoint & GRC \\
\hline \hline 0.8 & $-767226.75(1.83)$ & $-21592.04(3.72)$ & $-21175.49(3.76)$ \\
\hline 0.4 & $-401672.22(1.91)$ & $-5728.62(3.77)$ & $-5447.49(3.89)$ \\
\hline 0.2 & $-205683.40(1.95)$ & $-1497.58(3.83)$ & $-1358.81(4.01)$ \\
\hline 0.1 & $-104083.24(1.98)$ & $-445.40(3.36)$ & $-337.87(4.02)$ \\
\hline
\end{tabular}

TABLE 5.2. Error of the second moment for the Euler tau-leaping method (tauleap), the midpoint tau-leaping method (midpoint), and the GRC-tau-leaping method (GRC). The first column shows the time stepsize. The quantities outside of the parentheses from column 2 to 4 are the numerical errors with the corresponding $\tau$. The constants in the parentheses from column 2 to 4 show the ratio of the error in the upper row over the error in the current row (we do not show the results with $\tau=1.6$ ). The result implies that only Euler tau-leaping method is of first order, the other two methods are of second order for the second order moment.

\begin{tabular}{|c|c|c|c|}
\hline$\tau$ & tauleap & midpoint & GRC \\
\hline \hline 0.8 & $-1060.35(1.77)$ & $-501.42(1.97)$ & $-45.75(3.60)$ \\
\hline 0.4 & $-564.89(1.88)$ & $-252.36(1.99)$ & $-12.37(3.70)$ \\
\hline 0.2 & $-292.06(1.93)$ & $-124.94(2.02)$ & $-3.43(3.61)$ \\
\hline 0.1 & $-149.93(1.95)$ & $-63.04(1.98)$ & $-0.83(4.15)$ \\
\hline
\end{tabular}

TABLE 5.3. Error of the variance for the Euler tau-leaping method (tauleap), the midpoint tau-leaping method (midpoint), and the GRC-tau-leaping method (GRC). The first column shows the time stepsize. The quantities outside of the parentheses from column 2 to 4 are the numerical errors with the corresponding $\tau$. The constants in the parentheses from column 2 to 4 show the ratio of the error in the upper row over the error in the current row (we do not show the results with $\tau=1.6)$. The result implies that only GRC-tau-leaping method is of second order, the other two methods are of first order for the variance.

$\operatorname{Var}\left[X_{T}\right]=X_{0}\left(e^{2 c T}-e^{c T}\right)$. We simulate the system from $t=0$ to $T=11.2$ with fixed time-step $\tau$ and compute the numerical error at the final time $T=11.2$. The stepsize $\tau$ is chosen from 1.6 to 0.1 by halving one by one. Note that in this case

$$
\mathbb{E}\left(a_{0}\left(X_{t}\right) \tau\right) \geq 1000 \times 0.1 \times 0.1=10 \gg 1, \quad t \in[0, T],
$$

which means the tau-leaping condition is always satisfied during the simulation process. In order to demonstrate the order of accuracy, we follow a procedure that is widely used in the numerical study of ODEs. If the sample size is large enough, the statistical fluctuations in $E^{\tau}$ (the sample mean of $X_{T}$ with stepsize $\tau$ ) can be neglected. Then we double the stepsize to $2 \tau$ and obtain the sample mean $E^{2 \tau}$. If the simulation method has $p$-th order accuracy for the mean, there exists a constant $C$ such that

$$
\left|E^{\tau}-\mathbb{E} X\right| \approx C \tau^{p}
$$

It follows that

$$
R:=\frac{\left|E^{2 \tau}-\mathbb{E} X\right|}{\left|E^{\tau}-\mathbb{E} X\right|} \approx \frac{C(2 \tau)^{p}}{C \tau^{p}}=2^{p} .
$$

For example, if $p=2$ then $\left|E^{2 \tau}-\mathbb{E} X\right| \approx 4\left|E^{\tau}-\mathbb{E} X\right|$, i.e., the absolute error of the mean will be approximately four times larger when the stepsize is doubled. The ratios $R$ are shown in the parentheses in Tables 5.1, 5.2, and 5.3. 
Table 5.1, 5.2, and 5.3 list the errors of sample mean, second-order moment, and variance of $X_{T}$, respectively, with a sample size of $10^{8}$. We can see that the Euler tauleaping method is first order accurate for mean, second-order moment, and variance; midpoint tau-leaping is second-order accurate for the mean but first order accurate for the variance, which is consistent with the above analysis.

\section{Conclusion}

There are two scalings for the convergence analysis of tau-leaping methods in the literature $[1,30,26]$. We try to resolve this debate in this paper. We point out the shortcomings of both scalings. Theoretically we follow the large volume scaling in [1]. We find even in this framework that the midpoint tau-leaping does not improve the weak local order for the covariance compared with the explicit tau-leaping. We propose a procedure to explain the numerical order behavior by abandoning the dependence on the volume constant $V$ from the leading error term. The numerical examples validate this argument. We also generalize the global weak convergence analysis in [1] for any explicit tau-leaping type method in the large volume scaling, which can relieve the algorithm designer from the abstract analysis by just considering the local truncation errors. A rigorously proof of the convergence order of the covariance for these different methods is an interesting analysis problem to be considered in the future. Better algorithms to compute the covariance more exactly are needed.

Acknowledgement. The authors are supported by the National Science Foundation of China under grant 10871010. They also thank the referees for their constructive comments and suggestions to improve the paper.

Appendix A. Rooted tree theory for ODE. Consider a deterministic ODE in $d$ dimensional real space

$$
\frac{d \boldsymbol{x}(t)}{d t}=\boldsymbol{a}(\boldsymbol{x}(t)), \quad \boldsymbol{x}(0)=\boldsymbol{x}_{0} \in \mathbb{R}^{d} .
$$

The infinitesimal generator of this ODEs has the form

$$
\mathcal{L} f(\boldsymbol{x})=\lim _{t \rightarrow 0} \frac{f(\boldsymbol{x}(t))-f(\boldsymbol{x})}{t}=\sum_{i=1}^{d} a_{i}(\boldsymbol{x}) \partial_{i} f
$$

for any $f \in C^{1}\left(\mathbb{R}^{d}\right)$. In this section, we will use the Einstein summation convention to denote $\sum_{i=1}^{d} a_{i}(\boldsymbol{x}) \partial_{i} f$ as $a_{i} f^{i}$, where the superscript notation $f^{i}$ means the derivative of $f$ with respect to $x_{i}$.

The Taylor expansion of $f$ can be represented by the infinitesimal generator as

$$
f(\boldsymbol{x}(t+h)) \sim \sum_{n=0}^{\infty} \frac{h^{n}}{n !} \mathcal{L}^{(n)} f(\boldsymbol{x}(t)),
$$

where $\mathcal{L}^{(0)} f(\boldsymbol{x})=f(\boldsymbol{x})$ and $\mathcal{L}^{(n)} f(\boldsymbol{x})=\mathcal{L}\left(\mathcal{L}^{(n-1)} f(\boldsymbol{x})\right), n=1,2, \ldots$. From (A.2) we have

$$
\mathcal{L}^{(1)} f=a_{i} f^{i}, \quad \mathcal{L}^{(2)} f=a_{i} a_{j} f^{i j}+a_{j} a_{i}^{j} f^{i},
$$

and so on.

To analyze the Runge-Kutta methods for ODEs, Butcher developed the rooted tree theory which relates the Taylor series expansion in terms of combinations of elementary differentials which can be derived in a recursive fashion [6]. When $n=$ 


\section{0}

$n=0$
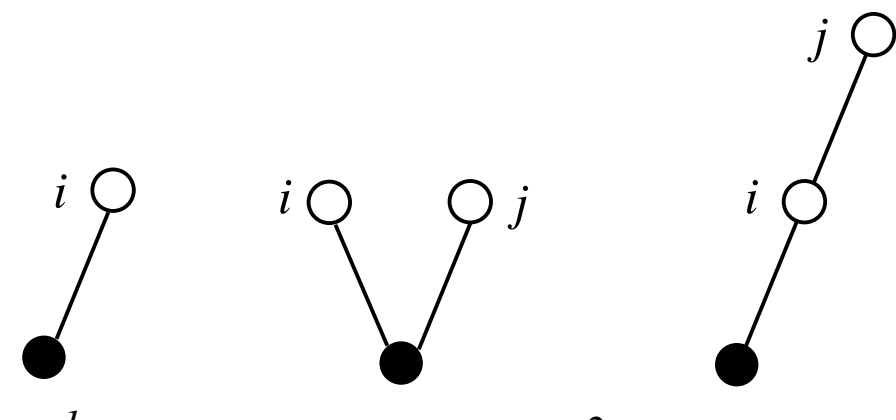

$n=2$

Figure A.1. The rooted tree representations of the elementary differentials for ODEs when $n=0,1,2$, where each tree corresponds to an element in $\mathcal{T}_{n+1}$. The solid circle represents the root node, which corresponds to the function $f$ in (A.4). The empty circle represents the leaf node, which corresponds to the coefficients a. The tree grows upwards when $n$ increases. The neighboring bottom-top pair nodes are assumed to have the father-son relation. If the node $i$ is the father of another node $j$, it means that there is a derivative with respect to the $j$-th variable for the coefficient $a_{i}$ corresponding to the node $i$.

$0,1,2$, the elementary differentials are shown in Figure A.1. Each time $n$ increases by 1 , one additional leaf node will be added into the tree. It can be connected into any existing node to form a new expanded tree. This property originates from the product rule of Newton calculus

$$
d(f g)=f \cdot d g+g \cdot d f
$$

which is valid for the infinitesimal generator (A.2). We define the rooted tree representation. Let $\mathcal{T}$ be the set of rooted trees and let $\boldsymbol{t}=\left[\boldsymbol{t}_{1}, \ldots, \boldsymbol{t}_{l}\right]$ be the tree formed by joining each $\boldsymbol{t}_{1}, \ldots, \boldsymbol{t}_{l}$ by a branch to a common root. Let $\boldsymbol{\epsilon}$ be the unique tree with 1 node, which takes the convention $l=0$. For the function $f \in C^{\infty}\left(\mathbb{R}^{d}\right)$, define the notation

$$
f(\boldsymbol{\epsilon})(\boldsymbol{x})=f(\boldsymbol{x})
$$

and the recursive definition of $f(\boldsymbol{t})(\boldsymbol{x})$ as

$$
f(\boldsymbol{t})(\boldsymbol{x})=f^{(l)}(\boldsymbol{x}) \cdot\left(\boldsymbol{a}\left(\boldsymbol{t}_{1}\right)(\boldsymbol{x}), \cdots, \boldsymbol{a}\left(\boldsymbol{t}_{l}\right)(\boldsymbol{x})\right),
$$

where the tensor $f^{(l)}(\boldsymbol{x})$ is the $l$-th order derivative of $f$. The right hand side of (A.7) means the multiplication of an $l$-th order tensor with respect to $l d$-vectors. For each $\boldsymbol{t}$, a number of useful combinatorial properties can be defined. $\rho(\boldsymbol{t}), \alpha(\boldsymbol{t})$ will denote, respectively, the number of nodes and the number of possible different monotonic labellings of $\boldsymbol{t}$ with root labeled first. If $\boldsymbol{t}=\left[\boldsymbol{t}_{1}, \ldots, \boldsymbol{t}_{l}\right]$, then they can be defined recursively as

$$
\left\{\begin{array}{l}
\rho(\boldsymbol{t})=1+\sum_{j=1}^{l} \rho\left(\boldsymbol{t}_{j}\right), \\
\alpha(\boldsymbol{t})=\left(\begin{array}{c}
\rho(\boldsymbol{t})-1 \\
\rho\left(\boldsymbol{t}_{1}\right), \ldots, \rho\left(\boldsymbol{t}_{l}\right)
\end{array}\right) \prod_{j=1}^{l} \alpha\left(\boldsymbol{t}_{j}\right) \frac{1}{\mu_{1} ! \mu_{2} ! \cdots}
\end{array}\right.
$$


and we take the following initial conditions and conventions:

$$
\rho(\boldsymbol{\epsilon})=1, \alpha(\boldsymbol{\epsilon})=1,0 !=1,\left(\begin{array}{c}
m \\
0
\end{array}\right)=1(m \in \mathbb{N}) .
$$

Here the integers $\mu_{1}, \mu_{2}, \ldots$ count the equal trees among $\boldsymbol{t}_{1}, \ldots, \boldsymbol{t}_{l}$ and the multinomial coefficient in the representation of $\alpha(\boldsymbol{t})$ counts the possible partitions of the labels $2, \ldots, \rho(\boldsymbol{t})$ to the $l$ trees. In addition, let $\mathcal{T}_{q}$ denote the set of all trees with $q$ nodes and $\mathcal{T}_{1}=\{\boldsymbol{\epsilon}\}$. Given these definitions and relationships, the following result holds:

Theorem A.1. The Taylor expansion (A.3) can be written as

$$
f(\boldsymbol{x}(t+h)) \sim \sum_{q=1}^{\infty} \sum_{\boldsymbol{t} \in \mathcal{T}_{q}} \frac{h^{\rho(\boldsymbol{t})-1}}{(\rho(\boldsymbol{t})-1) !} \alpha(\boldsymbol{t}) f(\boldsymbol{t})(\boldsymbol{x}) .
$$

For further details, the readers are referred to [20, 4]. ${ }^{2}$

Appendix B. Rooted tree theory for SDEs driven by Brownian motion. Suppose $\boldsymbol{X}_{t}$ satisfies the Ito SDEs driven by Brownian motion:

$$
d \boldsymbol{X}_{t}=\boldsymbol{a}\left(\boldsymbol{X}_{t}\right) d t+\boldsymbol{\sigma}\left(\boldsymbol{X}_{t}\right) \cdot d \boldsymbol{W}_{t},\left.\quad \boldsymbol{X}_{t}\right|_{t=0}=\boldsymbol{X}_{0} \in \mathbb{R}^{d},
$$

where $\boldsymbol{\sigma}: \mathbb{R}^{d} \rightarrow \mathbb{R}^{d \times m}, \boldsymbol{W}_{t}=\left(W_{1 t}, \ldots, W_{m t}\right)$ is an $m$-dimensional Wiener process. The infinitesimal generator of these SDEs has the form

$$
\mathcal{L} f(\boldsymbol{x})=\lim _{t \rightarrow 0} \frac{\mathbb{E}^{0, \boldsymbol{x}} f\left(\boldsymbol{X}_{t}\right)-f(\boldsymbol{x})}{t}=\left(a_{i} f^{i}+\frac{1}{2} b_{i_{1} i_{2}} f^{i_{1} i_{2}}\right)(\boldsymbol{x}),
$$

where the notation $\mathbb{E}^{0, \boldsymbol{x}}$ means the expectation with the initial state $\boldsymbol{x}$ at time $t=0$, and we have taken the definition that the matrix $\boldsymbol{B}=\boldsymbol{\sigma} \boldsymbol{\sigma}^{T}$. With Ito formula we have

$$
\mathbb{E}^{t, \boldsymbol{x}} f\left(\boldsymbol{X}_{t+h}\right) \sim \sum_{n=0}^{\infty} \frac{h^{n}}{n !} \mathcal{L}^{(n)} f(\boldsymbol{x}) .
$$

When $n=0,1,2$, the terms in the weak Taylor expansion are

$$
\begin{aligned}
\mathcal{L}^{(0)} f= & f, \quad \mathcal{L}^{(1)} f=a_{i} f^{i}+\frac{1}{2} b_{i_{1} i_{2}} f^{i_{1} i_{2}} \\
\mathcal{L}^{(2)} f= & a_{i} a_{j} f^{i j}+a_{j} a_{i}^{j} f^{i}+a_{i} b_{j_{1} j_{2}} f^{i j_{1} j_{2}}+\frac{1}{2} a_{j} b_{i_{1} i_{2}}^{j} f^{i_{1} i_{2}}+\frac{1}{2} b_{j_{1} j_{2}} a_{i}^{j_{1} j_{2}} f^{i} \\
& +b_{j_{1} j_{2}} a_{i}^{j_{2}} f^{i j_{1}}+\frac{1}{4} b_{i_{1} i_{2}} b_{j_{1} j_{2}} f^{i_{1} i_{2} j_{1} j_{2}}+\frac{1}{4} b_{j_{1} j_{2}} b_{i_{1} i_{2}}^{j_{1} j_{2}} f^{i_{1} i_{2}}+\frac{1}{2} b_{j_{1} j_{2}} b_{i_{1} i_{2}}^{j_{2}} f^{i_{1} i_{2} j_{1}} .
\end{aligned}
$$

To study the higher order Runge-Kutta methods for the weak approximation of the SDEs (B.1), Rössler proposed the colored rooted tree theory [31], in which the additional type of stochastic nodes is introduced except at the root node and at the deterministic nodes for the ODEs. Neglecting the coefficients for each term in (B.3), we can draw the labeled S-trees for the elementary differentials appearing in (B.2) and (B.3) in Figure B.1. Each time when $n$ increases by 1, one additional type of leaf

\footnotetext{
${ }^{2}$ Some of our representations are a bit different from those in [20,4] because we try to represent them in a unified way for ODEs, diffusion processes, and jump processes.
} 


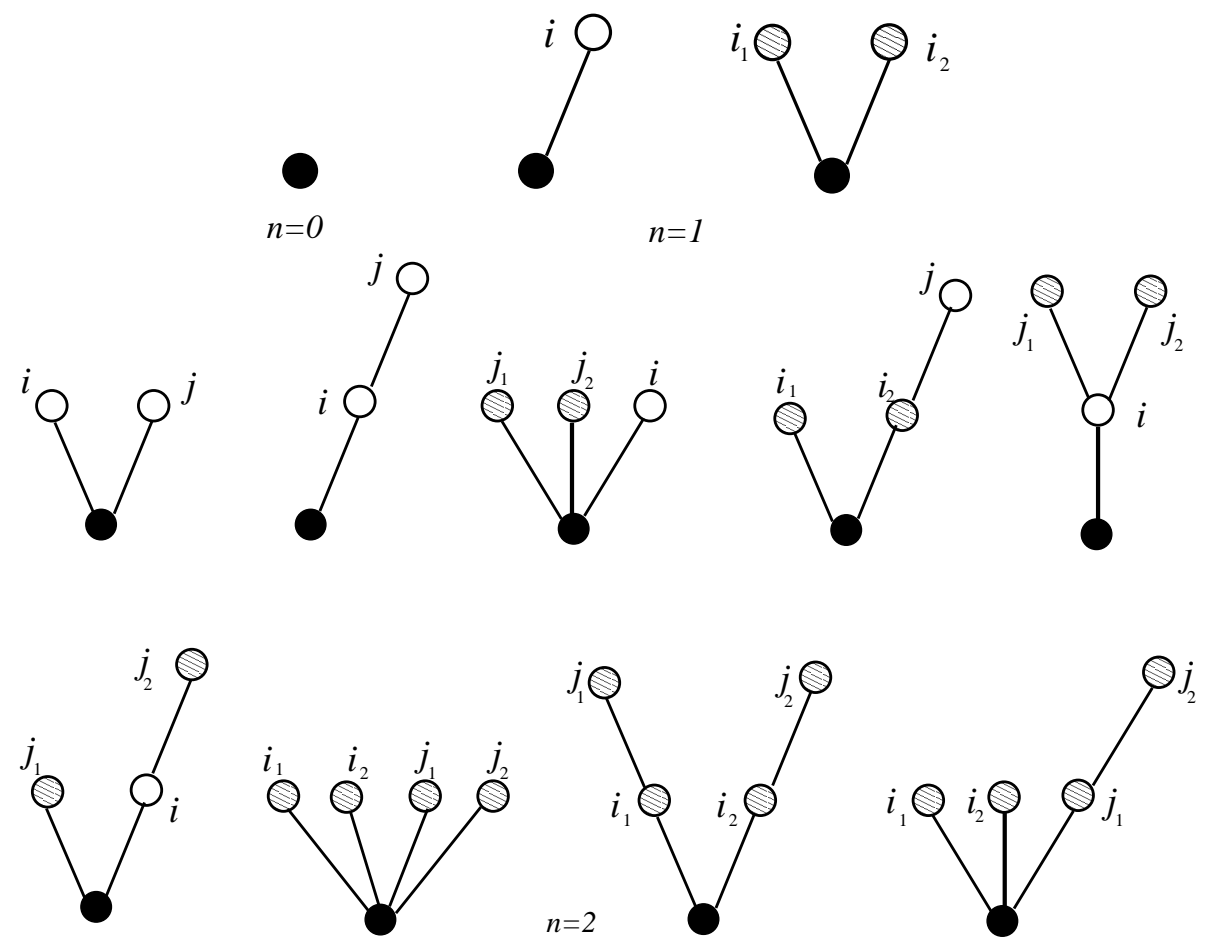

Figure B.1. The S-tree representations of the elementary differentials for SDEs driven by Brownian motion when $n=0,1,2$. The solid circle represents the root node, which corresponds to the function $f$ in (B.3). The empty circle represents the deterministic node, which corresponds to the coefficient functions $a$, and the shaded circle represent the stochastic node, which corresponds to the coefficients $b$. The tree grows upwards when $n$ increases. The neighboring bottom-top pair nodes are assumed to have the father-son relation. Similar to the ODE case, the father-son relationship means there is a derivative for the coefficient corresponding to the father node. Notice that the index with subindices like $i_{1}, i_{2}$, or $j_{1}, j_{2}$ corresponds to the integrated coefficient $b_{i_{1} i_{2}}$ or $b_{j_{1} j_{2}}$, but the derivatives are taken for their corresponding father nodes.

node (or nodes) will be added into the tree. They may be one deterministic node or two stochastic nodes, which is labeled by empty circles or shaded circles in Figure B.1, respectively. Then they are connected into the existing nodes to form a new expanded tree. The readers are referred to [31] for further information.

\section{REFERENCES}

[1] D. Anderson, A. Ganguly, and T. Kurtz, Error analysis of tau-leap simulation methods, Ann. App. Prob., accepted, 2010.

[2] A. Arkin, J. Ross, and H. McAdams, Stochastic kinetic analysis of developmental pathway bifurcation in phage $\lambda$-infected Escherichia coli cells, Genetics, 149, 1633-1648, 1998.

[3] A. Bortz, M. Kalos, and J. Lebowitz, A new algorithm for Monte Carlo simulation of Ising spin systems, J. Comp. Phys., 17, 10-18, 1975.

[4] K. Burrage and B.P.M., Conditions of Stochatic Runge-Kutta Methods by B-Series, SIAM J. Numer. Anal., 38, 1626-1646, 2001.

[5] K. Burrage and T. Tian, Poisson Runge-Kutta methods for chemical reaction systems, in Third International Workshop on Scientific Computing and Applications, City University of Hong Kong, 2003.

[6] J. Butcher, Coefficients for the study of Runge-Kutta integration processes, J. Austral. Math. 
Soc., 3, 185-201, 1963.

[7] Y. Cao, D. Gillespie, and L. Petzold, Avoiding negative populations in explicit Poisson tauleaping, J. Chem. Phys., 123, 054104, 2005.

[8] Y. Cao, D. Gillespie, and L. Petzold, Efficient stepsize selection for the tau-leaping method, J. Chem. Phys., 124, 44109, 2006.

[9] Y. Cao and L. Petzold, Trapezoidal tau-leaping formula for the stochastic simulation of biochemical systems, in Proceedings of the FOSBE 2005, University of California Santa Barbara, 2005.

[10] Y. Cao, L. Petzold, and D. Gillespie, The slow-scale stochastic simulation algorithm, J. Chem. Phys., 122, 14116, 2005.

[11] W. E, D. Liu, and E. Vanden-Eijnden, Nested stochastic simulation algorithm for chemical kinetic systems with disparate rates, J. Chem. Phys., 123, 194107, 2005.

[12] M. Elowitz, A. Levine, E. Siggia, and P. Swain, Stochastic gene expression in a single cell, Science, 297, 1183-1186, 2002.

[13] D. Endy and R. Brent, Modelling cellular behavior, Nature, 409, 391-395, 2001.

[14] M. Gibson and J. Bruck, Efficient exact stochastic simulation of chemical systems with many species and many channels, J. Phys. Chem. A, 104, 1876-1889, 2000.

[15] D. Gillespie, Stochastic simulation of chemical processes, J. Comput. Phys., 22, 403, 1976.

[16] D. Gillespie, Exact stochastic simulation of coupled chemical reactions, J. Phys. Chem., 81, 2340-2361, 1977.

[17] D. Gillespie, Approximate accelerated stochastic simulation of chemically reacting systems, J. Chem. Phys., 115, 1716, 2001.

[18] G. Gong and M. Qian, Lectures on Applied Stochastic Processes and the Stochastic Modeling in Algorithms and Intelligent Computing, Tsinghua University Press (in Chinese), Beijing, 2004.

[19] J. Gross and J. Yellen, Graph Theory and its Applications, CRC Press, Boca Raton, Florida, 2006.

[20] E. Hairer, S. Norsett, and G. Wanner, Solving Ordinary Differential Equations I: Nonstiff Problems, Springer, Berlin and Heidelberg, 1993.

[21] E. Haseltine and J. Rawlings, Approximate simulation of coupled fast and slow reactions for stochastic chemical kinetics, J. Chem. Phys., 117, 6959, 2002.

[22] Y. Hu and T. Li, Highly accurate tau-leaping methods with random corrections, J. Chem. Phys., 130, 124109, 2009.

[23] Y. Hu, T. Li, and B. Min, Local truncation error analysis of tau-leaping methods: Revisited, technical report 016, School of Mathematical Sciences, Peking University, Beijing, 2009.

[24] T. Kurtz, The relationship between stochastic and deterministic models for chemical reactions, J. Chem. Phys., 57, 2976-2978, 1972.

[25] T. Kurtz, Strong approximation theorems for density dependent Markov chains, Stoch. Proc. Appl., 6, 223-240, 1977/78.

[26] T. Li, Analysis of explicit tau-leaping schemes for simulating chemically reacting systems, Multi. Model. Simul., 6, 417, 2007.

[27] H. McAdams and A. Arkin, Stochastic mechanisms in gene expression, Proc. Natl. Acad. Sci. USA, 94, 814-819, 1997.

[28] S. Plyasunov, On hybrid simulation schemes for stochastic reaction dynamics, unpublished, 2005.

[29] M. Rathinam, L. Petzold, Y. Cao, and D. Gillespie, Stiffness in stochastic chemically reacting systems: The implicit tau-leaping method, J. Chem. Phys., 119, 12784, 2003.

[30] M. Rathinam, L. Petzold, Y. Cao, and D. Gillespie, Consistency and stability of tau-leaping schemes for chemical reaction systems, Multi. Model. Simul., 4, 867, 2005.

[31] A. Rössler, Stochastic Taylor expansions for the expectation of functionals of diffusion processes, Stoch. Anal. Appl., 22, 1553, 2004.

[32] A. Slepoy, A. Thompson, and S. Plimpton, A constant-time kinetic Monte Carlo algorithm for simulation of large biochemical reaction networks, J. Chem. Phys., 128, 205101, 2008. 